\title{
Strongly Coupled Assimilation of a Hypothetical Ocean Current Observing Network within a Regional Ocean-Atmosphere Coupled Model: An OSSE Case Study of Typhoon Hato
}

\author{
Luke Phillipson, ${ }^{\mathrm{a}}$ Yi Li, ${ }^{\mathrm{b}, \mathrm{c}, \mathrm{d}}$ AND RALF TOUMI ${ }^{\mathrm{a}}$ \\ ${ }^{a}$ Space and Atmospheric Physics, Department of Physics, Faculty of Natural Sciences, Imperial College London, London, \\ United Kingdom \\ ${ }^{\mathrm{b}}$ Key Laboratory of Marine Hazards Forecasting, Ministry of Natural Resources, Hohai University, Nanjing, China \\ ${ }^{\mathrm{c}}$ College of Oceanography, Hohai University, Nanjing, China \\ ${ }^{\mathrm{d}}$ State Key Laboratory of Satellite Ocean Environment Dynamics, Second Institute of Oceanography, Ministry of Natural Resources, \\ Hangzhou, China
}

(Manuscript received 6 April 2020, in final form 15 January 2021)

\begin{abstract}
The forecast of tropical cyclone (TC) intensity is a significant challenge. In this study, we showcase the impact of strongly coupled data assimilation with hypothetical ocean currents on analyses and forecasts of Typhoon Hato (2017). Several observation simulation system experiments (OSSE) were undertaken with a regional coupled ocean-atmosphere model. We assimilated combinations of (or individually) a hypothetical coastal current HF radar network, a dense array of drifter floats, and minimum sea level pressure. During the assimilation, instant updates of many important atmospheric variables (winds and pressure) are achieved from the assimilation of ocean current observations using the cross-domain error covariance, significantly improving the track and intensity analysis of Typhoon Hato. Relative to a control experiment (with no assimilation), the error of minimum pressure decreased by up to $13 \mathrm{hPa}(4 \mathrm{hPa} / 57 \%$ on average $)$. The maximum wind speed error decreased by up to $18 \mathrm{kt}(5 \mathrm{kt} / 41 \%$ on average) $\left(1 \mathrm{kt} \approx 0.5 \mathrm{~m} \mathrm{~s}^{-1}\right)$. By contrast, weakly coupled implementations cannot match these reductions $(10 \%$ on average $)$. Although traditional atmospheric observations were not assimilated, such improvements indicate that there is considerable potential in assimilating ocean currents from coastal HF radar and surface drifters within a strongly coupled framework for intense landfalling TCs.
\end{abstract}

KEYWORDS: Atmosphere-ocean interaction; Tropical cyclones; Numerical weather prediction/forecasting; Coupled models; Data assimilation; Regional models

\section{Introduction}

Tropical cyclones (TC) are challenging to forecast. With an increasing global population density around coastal cities (Neumann et al. 2015) (such as the Pearl River Delta) and projected more intense hurricanes under climate change scenarios (Ting et al. 2019), continued improvements to the forecast skill is essential. Over the past few decades, track forecasts have steadily improved (Gall et al. 2013). Intensity predictions have also improved but at a much slower pace (DeMaria et al. 2014; Cangialosi et al. 2020), and remain highly challenging for rapidly intensifying TCs. Crucial model components for intensity forecasts include correctly capturing the initial vortex structure, moisture content and air-sea fluxes (Zhang and Emanuel 2018). For improving the initial model vortex the use of data assimilation (DA; Lahoz and Schneider 2014) is well established (e.g., Weng and Zhang 2012). Two broad classes of DA algorithms exist; variational and sequential. These classes have been typically implemented as either 4D variational data assimilation (4D-Var) (Talagrand and Courtier 1987) or as the ensemble Kalman filter (EnKF) (Evensen 1994). For TC forecasting, the benefit of assimilating atmospheric observations (satellite and in situ) within a 4D-Var or EnKF system has been extensively studied

Corresponding author: Yi Li, yli.ouc@gmail.com (e.g., Pu et al. 2019; Yang et al. 2016; Zhang and Weng 2015; Aksoy et al. 2012; Liu et al. 2012; Torn and Hakim 2009; Zhang et al. 2009).

Ocean-atmosphere coupled general circulation models (CGCMs) have also shown significant improvements for TC forecast by incorporating air-sea fluxes and ocean feedbacks (e.g., Hodur 1997; Sandery et al. 2010; Holt et al. 2011; Doyle et al. 2012; Yihong et al. 2013; Zambon et al. 2014; Na et al. 2015; Srinivas et al. 2016; Mogensen et al. 2017; Flaounas et al. 2018; Lengaigne et al. 2019; Zhang and Emanuel 2018). Implementing DA into CGCMs was initially developed as weakly coupled DA (WCDA) (e.g., Saha et al. 2006; Bender et al. 2007; Zhang et al. 2007; Kunii et al. 2017; Karspeck et al. 2018; Laloyaux et al. 2018). WCDA only updates state variables belonging to one model component in the coupled system. The coupler then passes information (such as the air-sea fluxes) to and from each respective model during the forward integration.

Very few studies have directly investigated the impact of assimilating ocean observations on TC forecasts in a coupled system (Domingues et al. 2019). Using an observing system simulation experiment (OSSE) framework, Dong et al. (2017) investigated the impact of hypothetical underwater glider assimilation (temperature and salinity profiles) compared to a standard suite of ocean observations (altimetry and sea surface temperature) in WCDA system. They found that the assimilation of glider observations alone did not have an impact 
on the intensity forecast due to their limited coverage. The combined assimilation of the standard ocean observations and glider temperature/salinity $(T / S)$ profiles did, however, moderately improve the TC intensity forecast. This was attributed to a better representation of the barrier layer and ocean stratification.

A more attractive variant to WCDA is regarded as strongly coupled DA (SCDA) (Penny and Hamill 2017). SCDA systems have one DA system that encompasses both models, updating all state variables across model domains instantaneously. This methodology has been utilized for both weather and climate models (Smith et al. 2015; Frolov et al. 2016; Laloyaux et al. 2016a,b; Penny and Hamill 2017; Laloyaux et al. 2018; Magnusson et al. 2019; Penny et al. 2019), often giving superior results over the weakly coupled alternative.

Li and Toumi (2018) recently developed an SCDA system with an ensemble adjustment Kalman filter (EAKF) for a regional convection-permitting ocean-atmosphere model. In an idealized TC setup, Li and Toumi (2018) showed, for the first time, that the assimilation of ocean currents could provide instant updates of TC winds via the prior cross-domain sample covariance, subsequently improving TC forecasts. A robust physical correlation between ocean currents and TC wind speeds has been previously observed (Chang et al. 2016). This allows for a simplified implementation of the localization function between model components, as the length and temporal scales of the surface ocean currents and the atmospheric boundary are closely linked under high winds.

$\mathrm{Li}$ and Toumi (2018) envisaged how coastal high-frequency (HF) radars observing the surface ocean currents could provide hourly surface/boundary layer updates. This is an advantage over less frequent satellite observations. Furthermore, atmospheric radars do not directly observe the critical surface winds. Chen and Zhang (2019) recently expanded on Li and Toumi (2018) with a case study of the rapid weakening event in Hurricane Florence (2018). Using an OSSE framework, they assimilated a highly idealized uniform network of sea surface temperature (SST), sea surface height (SSH) and ocean current velocities $(U / V)$. They confirmed Li and Toumi (2018) findings that the assimilation of surface currents could improve the hurricane analysis and forecast.

In this study, we explore further the potential of an SCDA system with a hypothetical ocean observing network in the South China Sea (SCS) and a case study of a rapidly intensifying landfalling TC, Typhoon Hato (2017). Typhoon Hato formed as a tropical depression over the east of Luzon on 19 August 2017 and became a category-3 typhoon before making landfall over Zhuhai, Guangdong, at 0450 UTC 23 August 2017. As Typhoon Hato advanced into the SCS, it underwent rapid intensification $(\mathrm{RI})$ of $10 \mathrm{kt}\left(1 \mathrm{kt} \approx 0.5 \mathrm{~m} \mathrm{~s}^{-1}\right)$ within just 3 h (Pun et al. 2019). This RI close to landfall meant Typhoon Hato was very challenging to forecast. Macau was especially impacted and experienced unprecedented levels of flooding (Li et al. 2018). In total, USD 7 billion of damage was estimated, accompanying 22 fatalities (Zhao et al. 2020). The Pearl River Delta has a population of 60 million and, according to the World Bank, is the largest urban megacity in the world (World Bank 2015). Its GDP is now more than USD 1.2 trillion, and it hosts some of the world's major ports and global supply chains. At the same time, the delta experiences on average, five major storms per year (Chao and Chao 2012). No other region in the world has such a combination of economic exposure and frequency of landfall. Currently, this region does not have the ocean current observation network proposed in this paper. However, we will show that such a network could bring improvements to TC predictions, and hence potential economic benefits.

In a recent review Domingues et al. (2019) advocated the investigation of ocean observation networks for TC forecasts, noting the potential of targeted ocean observations ahead of the TC (gliders, profiling floats, drifters, etc.). Therefore, our hypothetical observation network will include a vast HF radar network and numerous drifters "deployed" ahead of the TC in a proof-of-concept idealized deployment pattern. Drifter observations have been assimilated in several studies on ocean forecasting (Molcard et al. 2003; Özgökmen et al. 2003; Molcard et al. 2005; Nodet 2006; Salman et al. 2006; Nilsson et al. 2012; Carrier et al. 2014; Muscarella et al. 2015; Coelho et al. 2015; Carrier et al. 2016; Phillipson and Toumi 2017; Sun and Penny 2019) but not yet explored in the context of SCDA and TC forecasts (Domingues et al. 2019).

In the next section, we describe the coupled model, data assimilation system, and the experiments to be performed. Then the hypothetical observation network and "Truth"/control simulations are examined. We then analyze the impact of the ocean current assimilation on the TC. We last explore the impact on several forecasts.

\section{Materials and methods}

\section{a. Numerical models}

The Coupled Ocean-Atmosphere-Wave-Sediment Transport (COAWST v3.3) Modeling System (Warner et al. 2010) encompasses several models. In this study, the coupled framework is composed only of the Regional Ocean Modeling System (ROMS v3.7) and the Advanced Research version of the Weather Research and Forecasting Model (WRFARW v3.9.1.1).

ROMS is a hydrostatic, primitive equation, Boussinesq ocean general circulation model as described by Shchepetkin and McWilliams (2005). The model domain extends over $10.6^{\circ}-28.1^{\circ} \mathrm{N}$ and $110.2^{\circ}-121.2^{\circ} \mathrm{E}$, and is rotated counterclockwise by $20^{\circ}$ (latitude axis) to maximize the ocean coverage. The horizontal resolution is $4 \mathrm{~km}$, with 20 terrain-following vertical levels. ROMS $\sigma$ vertical compression parameters (controlling the degree of enhanced resolution at the surface and bottom boundary layer) are $h_{c}=250 \mathrm{~m}, \sigma_{s}=5$, and $\sigma_{b}=0.4$. The vertical levels are compressed within the first $250 \mathrm{~m}$ of the surface, increasing the vertical resolution of the surface currents. Lateral boundary and initial conditions for temperature, salinity, ocean current velocities, and sea surface height were obtained from the Global Ocean Forecasting System, version 3.1 (GOFS 3.1), 41-layer HYCOM + NCODA Global $1 / 12^{\circ}$ Analysis obtained from the National Ocean Partnership Program (https://www.hycom.org/dataserver/ gofs-3pt1/analysis). 
WRF-ARW is a nonhydrostatic model of the fully compressible Euler equations as described extensively in Skamarock et al. (2008). The model domain extends over $5^{\circ}-30^{\circ} \mathrm{N}$ and $103.5^{\circ}-$ $130^{\circ} \mathrm{E}$. The horizontal resolution is $6 \mathrm{~km}$, with 40 vertical levels. The highest level extends to $50 \mathrm{hPa}$. The WRF single-moment 6-class scheme (Hong and Lim 2006) is used for the microphysics. The RRTMG shortwave and longwave schemes (Iacono et al. 2008) are used for the radiative forcing. The revised MM5 scheme (Jiménez et al. 2012) is used for the surface layer physics with the additional air-sea surface flux parameterization for high wind conditions [option one Green and Zhang (2013)]. The Yonsei University scheme is used for planetary boundary layer parameterization (Hong et al. 2006). Kain-Fisher cumulus parameterization (Kain 2004) with a modified convective trigger function (Ma and Tan 2009) was used. Lateral boundary and initial conditions are provided by the NCEP Global Forecast System at $0.25^{\circ}$ resolution (National Centers for Environmental Prediction/National Weather Service/NOAA/U.S. Department of Commerce 2015).

The Model Coupling Toolkit (MCT) distributes fluxes to/from each model component (Larson et al. 2005). From WRF, the wind stress, radiation (longwave and shortwave), sea level pressure, and heat fluxes (latent and sensible) are transferred to ROMS. From ROMS, only the sea surface temperature is transferred to WRF. The coupling exchange frequency was set to 1 per $1 \mathrm{~min}$.

\section{b. Assimilation scheme: DART-EAKF under a local least squares framework}

The data assimilation algorithm is the same as in Li and Toumi (2018), the ensemble adjustment Kalman filter (Anderson 2001) under a two-step local least squares framework (Anderson 2003). This algorithm is utilized within COAWST via the Data Assimilation Research Testbed (DART, version Lanai) software (Anderson et al. 2009). DART is an open-source, modular community portable software utilized for a wide variety of models (e.g., Raeder et al. 2012; Hoteit et al. 2013; Romine et al. 2013; Li and Toumi 2017; Aydoğdu et al. 2018; Singh 2020). The first step to the algorithm is finding the update increment in observation space $\Delta y_{n}$, computed for each $n$ prior ensemble estimate of the observation variable $y$. This update is achieved by applying a scalar ensemble filter (such as the EAKF) to each observation. Once observation-space increments $\Delta y_{n}$ have been computed, the second step is to apply a least squares fitting that distributes the observational space increments $\Delta y_{n}$ to each model state vector $x_{m}$ for each $n$ ensemble member across each 3D model grid point $i, j, z$ :

$$
\Delta x_{m, n, i, j, z}=\frac{\sigma\left(x_{m, i, j, z}, y\right)}{\sigma(y, y)} \Delta y_{n},
$$

where $\Delta x_{m, n, i, j, z}$ is the update increment for ensemble member $n$ and model variable $m$ at the longitude $i$, latitude $j$ and vertical $z$ grid point, $\Delta y_{n}$ is the observation-space increment, $\sigma\left(x_{m, i, j, z}, y\right)$ is the prior sample covariance between prior ensemble estimates of the observation variable $y$ and each model state vector $x_{m, i, j, z}$, and $\sigma(y, y)$ is the prior variance of observation variable $y$.
This two-step framework has many advantages, such as computational efficiency and parallel implementation, while providing identical results (to computational round-off) as compared to standard implementations of the EAKF (Anderson 2003). Furthermore, strongly coupled data assimilation is simple to implement within such a framework. If prior sample covariances across both models are available, then the observational increment computed from one model can be regressed onto the other. The state variables $x_{m}$ need only include ocean and atmospheric variables.

For our COAWST-DART system, as in Li and Toumi (2018), $x_{m}$ is extended to include variables across both models. For any observation that is assimilated, the following WRF variables are updated; 3D velocity, temperature, pressure, geopotential height, and mixing ratios of water vapor, rainwater, cloud water, graupel, ice and snow. While in ROMS, the 3D horizontal currents, salinity, temperature and sea surface height are updated. Figure 1 summarizes the key steps to the COAWST-DART SCDA system.

While it may be trivial to implement SCDA in COAWSTDART, the challenge lies in correctly defining the prior error covariance localization between the ocean and atmosphere variables, an area of ongoing research in SCDA [Smith et al. 2015; Sluka et al. 2016; Z. Shen et al. 2018, unpublished manuscript (https://doi.org/10.5194/npg-2018-50); Smith et al. 2018; Yoshida and Kalnay 2018; Penny et al. 2019]. Covariance localization is a critical part of ensemble DA systems in order to mitigate spurious correlations from moderate ensemble sizes (Houtekamer and Mitchell 1998). With SCDA, the problem is made even more complicated with the addition of crossdomain variables to the prior error covariance. The complexity arises since the atmosphere and ocean contain very different temporal and spatial scales, allowing spurious correlations to infiltrate the posterior update more easily with suboptimal definitions (Zhang et al. 2020).

For our application, the temporal and spatial scales of the assimilated surface ocean currents are almost entirely driven by the TCs from the atmosphere. Under such strong wind conditions, we can assume the scales of the surface ocean currents and atmosphere can approximately collapse to a single scale (i.e., the scale of the atmosphere driving the ocean currents). The inherent connection between the ocean surface and the upper atmosphere for our TC driven application mitigates the need for a more refined approach to cross-model localization. Nevertheless, we note that the spatial and temporal scales of other oceanic variables and atmospheric exchanges (such as SST cooling feedback) are present. Therefore, this one-scale approach is limited to a specific proof-of-concept of surface current assimilation for TCs ( $\mathrm{Li}$ and Toumi 2018). Future studies will consider the implementation of different horizontal/vertical localization scales for each model component and across domains [e.g., Smith et al. 2015; Sluka et al. 2016; Frolov et al. 2016; Z. Shen et al. 2018, unpublished manuscript (https://doi.org/10.5194/npg-2018-50); Smith et al. 2018; Yoshida and Kalnay 2018; Penny et al. 2019].

Therefore, for the vertical localization within WRF (and the cross-domain ROMS to WRF), a single Gaspari-Cohn localization function (Gaspari and Cohn 1999) with a vertical 


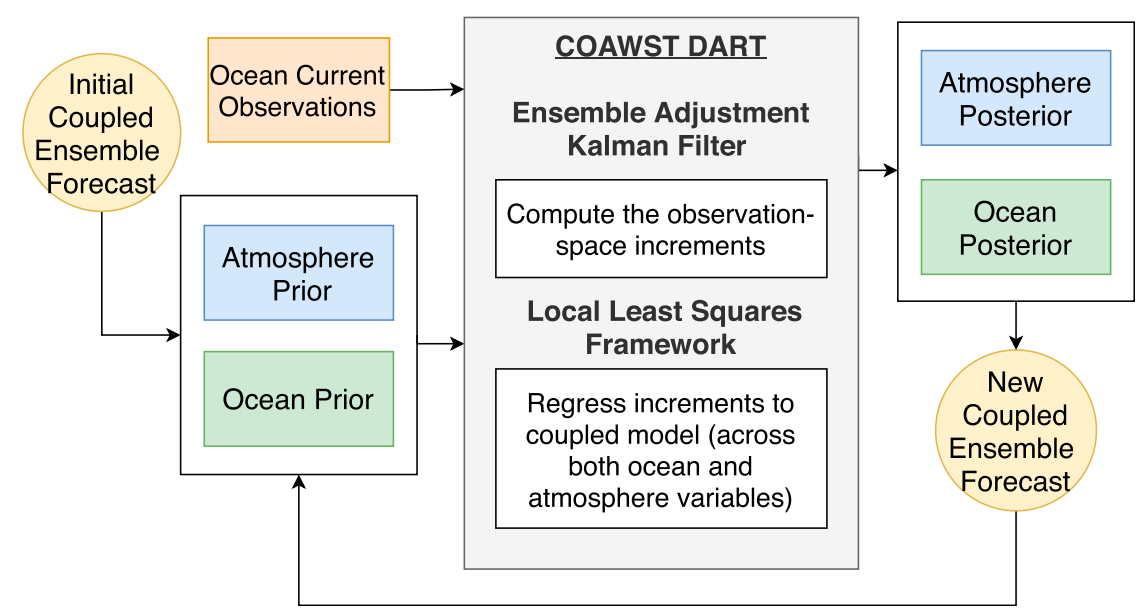

FIG. 1. A flowchart depicting the procedure of the COAWST-DART system from the initial coupled ensemble forecast to the updated forecast.

half-width length scale at $20 \mathrm{~km}$ was implemented. This choice reflects the influence of the surface ocean currents vertically into the atmosphere. Green and Zhang (2013) suggested high correlations $(>0.6)$ between the lower-level winds and above can extend as high as $20 \mathrm{~km}$ within the TC core. After a series of sensitivity simulations and an informal investigation of correlations (not shown), we chose a half-width radius covering the full atmospheric column $(20 \mathrm{~km})$. This scale helped achieve a smooth vertical adjustment that penetrates deep into the atmosphere when assimilating currents directly underneath the inner circulation of Hato. This single localization function is used throughout the WRF domain whether the observations are inside or outside the TC core.

Vertical localization within ROMS has not yet been implemented within the COAWST-DART system, and therefore, the whole water column is impacted by the surface assimilation equally. This limitation is likely to cause spurious correlations between the ocean surface and at depth. However, any potential sampling error deeper in the ocean model is unlikely to impact the surface currents in the next cycle. This is because of the much longer time scales exhibited by the deep ocean (days) as compared to the hourly frequency of each assimilation cycle. Furthermore, the initial spread in the deeper ocean is near zero (owing to the surface forcing driving the spread) and will therefore provide a limited contribution to the surface update.

The horizontal half-width radius for WRF, ROMS and cross-domain assimilation was set equally as $150 \mathrm{~km}$. This scale was chosen to be approximately the radius of gale-force winds (R18) of western North Pacific Ocean typhoons, $\sim 250 \mathrm{~km}$ (Sampson et al. 2017). Observation errors for the HF radar currents and drifters currents are both set to $10 \mathrm{~cm} \mathrm{~s}^{-1}$ ( $\mathrm{Li}$ and Toumi 2018). The observation error for the minimum pressure dataset was set to $8 \mathrm{hPa}$. A total of 34 ensemble members were used in the system, a computational compromise at the expense of the overall cost of running a complex coupled ensemble system. To improve the spread, spatially and temporally adaptive inflation (Anderson 2007, 2009), was also applied with standard settings among the WRF-DART community (Ha and Snyder 2014).

The initial ensemble was created as in Torn and Hakim (2008), Meng and Zhang (2011), Li and Liu (2009), Liu and Li (2010), and He et al. (2019). First, the WRF initial and boundary conditions at 1200 UTC 21 August for each ensemble member are perturbed by adding random draws from balanced background error covariance statistics of the WRF $3 \mathrm{D}$ variational data assimilation (3D-VAR) system [option 3 of control variables (CV3), or background option; Barker et al. 2004]. The perturbed variables include the horizontal winds, temperature, and mixing ratio for water vapor. The perturbed WRF ensemble is then used in the coupled COAWST system, which is integrated for $6 \mathrm{~h}$ (until 1800 UTC 21 August), developing divergence in TC trajectories between ensemble members. The ROMS initial conditions at 1200 UTC 21 August are not perturbed. In an ensemble DA system for the ocean, the initial spread is typically created by either generating random perturbations (Evensen 1994; Brusdal et al. 2003; Wan et al. 2008; $\mathrm{Li}$ and Toumi 2017), taking random snapshots of the model state at different integration times (Leeuwenburgh 2005; Garcia et al. 2016) or through driving each ensemble member with a perturbed atmospheric reanalysis (Penny et al. 2015). For this TC application, sufficient spread in the surface ocean currents can be achieved through the spread of TCs within the ensemble that develops during the $6 \mathrm{~h}$ integration of the coupled model.

\section{c. Observing system simulation experiment}

OSSEs are performed to assess whether assimilating a candidate observation network could improve the track and intensity of Typhoon Hato. Hypothetical observations (an HF radar network, drifter array, and minimum TC pressure) are extracted from an initial coupled stimulation of Typhoon Hato (Truth). These synthetic "observations" are subsequently assimilated into the model system and compared with a simulation without any assimilation (CNTRL).

The coupled simulation forming the Truth was created similarly to Chen and Zhang (2019). For their case study on 


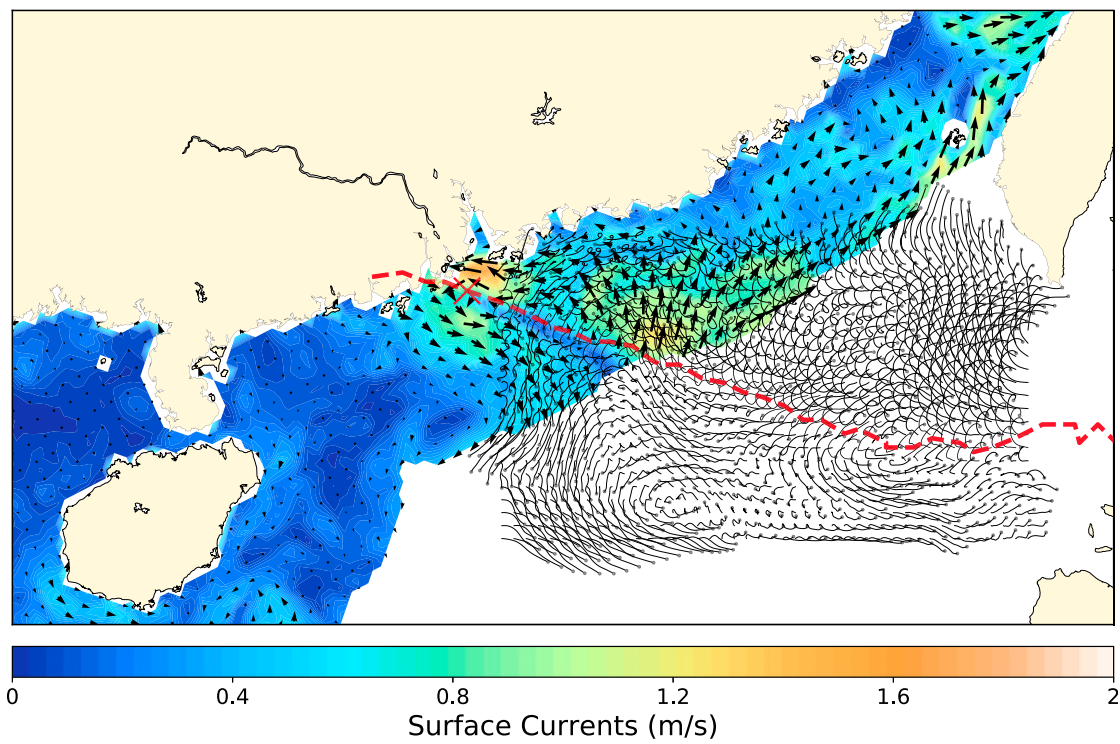

FIG. 2. The hypothetical observation network of the surface ocean currents at peak intensity within the Truth simulation. The HF radar network is depicted in color, with the direction of the currents overlaid in black vectors. The drifter dataset is depicted with black lines following the position of each "deployed drifter" up to the current position (black dot). The red X depicts the location of the typhoon center. The red line depicts the track for the entire Truth simulation.

Hurricane Florence, Chen and Zhang (2019) performed an initial 21-member CNTRL ensemble forecast and selected their truth as the member with the smallest intensity and track errors. However, if we did this, a clear landfall ocean current bias remained (temporally and spatially). Therefore, we modified the approach of Chen and Zhang (2019) by instead running our COAWST-DART system, assimilating hourly best-track TC position, minimum pressure, and maximum wind speed (e.g., Chen and Snyder 2007; Torn and Hakim 2009; Zhang et al. 2014). The best-track data of Typhoon Hato was obtained from the Joint Typhoon Weather Centre (JTWC). Small observational errors $(25 \mathrm{~km}$ for vortex position, $8 \mathrm{hPa}$ for minimum pressure, and $1.6 \mathrm{~m} \mathrm{~s}^{-1}$ for maximum wind speed) were used in the DA system to constrain our TC strictly around the dataset. From the resulting posterior ensemble, the member that best matched the JTWC data was then used as the verifying Truth. We later demonstrate that the verifying Truth is a superior representation of Typhoon Hato as compared to any of the CNTRL ensemble members. Three distinct datasets are constructed from the verifying Truth.

The proposed HF radar network has complete coverage of ocean currents along the entire coastline available every hour (Fig. 2). This is created by interpolating the hourly Truth surface currents onto a $12 \mathrm{~km}$ uniform grid restricted to up to $200 \mathrm{~km}$ from the coastline. This resolution and range are typically achievable by HF radar systems (Paduan and Washburn 2013; Mantovani et al. 2020). The resulting velocities $(u, v)$ are assimilated at this resolution $(12 \mathrm{~km})$ with no data thinning. As far as the authors are aware, no such network currently extends for the entirety of Southern China (Roarty et al. 2019). However, a handful of radars are operational along southern
China (Fujii et al. 2013) providing some irregular coverage. Additionally, Taiwan contains up to 18 radars that also partially sample the SCS (Shen et al. 2019). Such observations could, therefore, be utilized in future studies with real-time TC events.

The proposed drifter array consists of 1000 drifters "deployed" in a uniform square (16-km effective resolution) ahead of Typhoon Hato (black lines in Fig. 2). This hypothetical deployment is a proof-of-concept to demonstrate the potential benefits. The initial drifter positions are then advected by the Truth surface currents using OceanParcels v2.0.0 (Delandmeter and van Sebille 2019). The center finite difference of drifter hourly positions generates hourly horizontal ocean current velocities $(u, v)$ that are directly assimilated.

The minimum pressure of the Typhoon Hato within the Truth simulation is extracted every $6 \mathrm{~h}$. This dataset is intended to replicate real-time TC guidance products that are often assimilated by operational centers (Kleist 2011). Similar to Heming (2016) this 6-hourly product is then linearly interpolated to an hourly dataset. Informal experiments (not shown) found the assimilation of the minimum pressure at a 6-hourly interval did not effectively constrain the TCs in the ensemble.

Seven OSSEs are undertaken. The first three consist of the individual assimilation of each dataset: the HF radar network currents (HF), the drifter currents (DRIFT), and the minimum pressure dataset (PMIN). The next two are the combined assimilation of $\mathrm{HF}$ radar network and drifter currents $($ HF + DRIFT) and additionally combined with the minimum pressure dataset $(\mathrm{PMIN}+\mathrm{HF}+\mathrm{DRIFT})$. The final two are weakly coupled variants of the combined assimilation experiments (HF+DRIFT WC and PMIN+HF+DRIFT WC). For 
HF+DRIFT WC, $X_{m}$ in Eq. (1) is reduced to only the ROMS variables. For PMIN $+\mathrm{HF}+\mathrm{DRIFT} \mathrm{WC}, X_{m}$ is adapted so that observational space increments $\Delta y_{n}$ for one particular model component (say PMIN) can only impact the same model (i.e., WRF). There is no cross model component for either WC experiment. Each dataset is assimilated using a 1-h analysis cycle throughout a 36-h simulation starting from 1800 UTC 21 August. The same initial ensemble is used for all experiments created from a short 6-h coupled run as previously described.

The root-mean-square difference (RMSD) between the simulated currents in the prior and posterior ensemble (before and assimilation) and the Truth simulation is computed to evaluate the performance of the COAWST-DART assimilation. The spread of the ensemble is also computed as calculated in Fortin et al. (2014). We then assess the TC minimum pressure (Pmin), maximum wind speed (Vmax), the radius of galeforce $\left(18 \mathrm{~m} \mathrm{~s}^{-1}\right)$ wind (R18), integrated power dissipation (IPD), and wind field and track errors. The integrated power dissipation (IPD) is defined as in Wang and Toumi (2018). We also investigate the impact on the ocean state, including the sea surface height ( $\mathrm{SSH}$ ), temperature (SST) and sea surface salinity (SSS). Last, we perform a suite of forecasts (free-running COAWST simulations without any DA) from five different initialization times for three experiments (PMIN, PMIN+HF+DRIFT, and PMIN+HF+DRIFT WC). We compare the relative accuracy of these forecasts (as compared with the Truth TC) for all TC metrics.

\section{Results}

\section{a. Truth and control simulation}

Hato metrics are well captured by the Truth simulation (red dashed line in Fig. 3). For example, over the entire simulation, the root-mean-square error (RMSE) of Pmin and Vmax is $1.3 \mathrm{hPa}$ and $8 \mathrm{kt}$, respectively. The track is well reproduced, with an RMSE of $17 \mathrm{~km}$ in the last $24 \mathrm{~h}$ before landfall. However, the assimilation of vortex variables (location, Pmin, and Vmax) cannot constrain the outer circulation (R18). The constraint on Vmax is also noticeably more variable than that of Pmin, with relatively larger fluctuations (often overestimating Vmax) in the time series evolution (Fig. 3b). Our Truth simulation of Typhoon Hato, therefore, has a smaller scale and is more intense than the best-track data. However, despite these differences, the Truth simulation crucially produces an associated ocean current field response at the correct time and location. The TC within the simulation also undergoes RI that approximately matches the best-track data (Fig. 3).

The CNTRL ensemble represents the evolution of the same initial ensemble as used in the DA experiments, but without any assimilation (green box and whiskers/lines in Fig. 3). For this CNTRL, the majority of members underestimate the Pmin and Vmax. This underestimation could be due to the limited resolution of the WRF Model $(6 \mathrm{~km})$, and the large spatial spread of the cyclones within the ensemble (Fig. 3d). Furthermore, while the ensemble average cross-track error is small, the along-track error is considerable (Fig. 3d). The translation speed of Typhoon Hato is not well replicated. Compared to our Truth simulation (with best-track DA), the CNTRL ensemble does not adequately

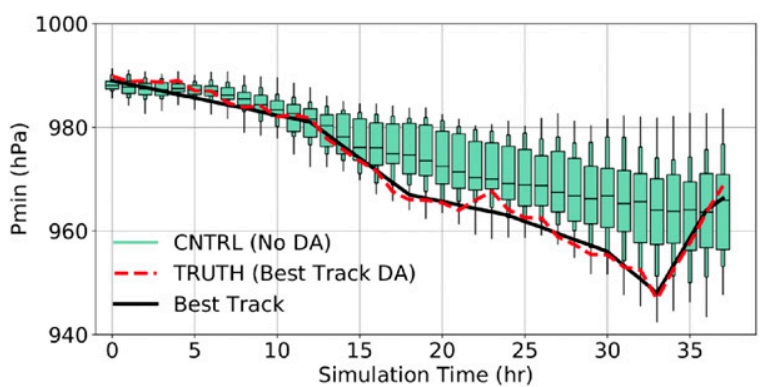

a)

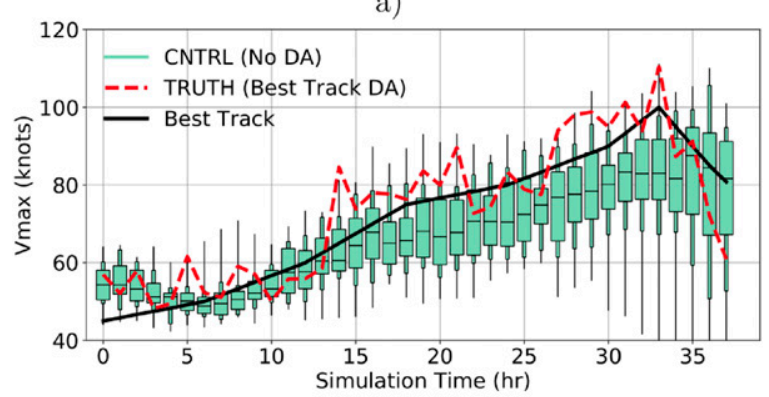

b)

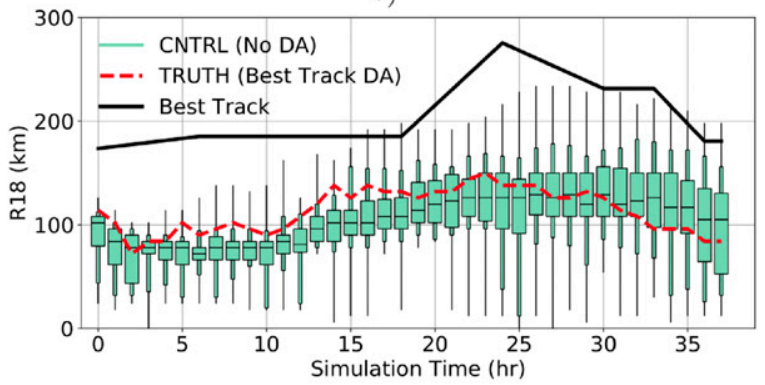

c)

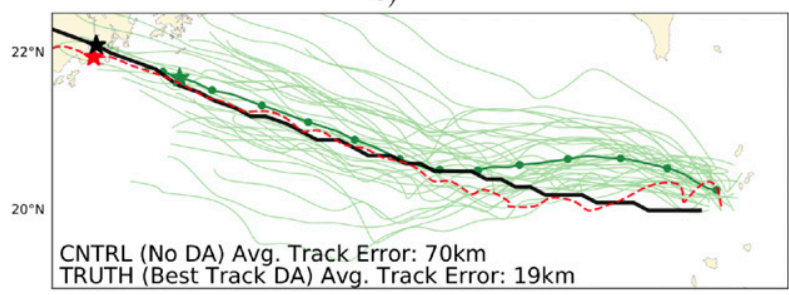

d)

FIG. 3. (a) The ensemble minimum pressure, (b) maximum wind speed, (c) gale-force radius (R18) time series and (d) track. The box-and-whisker plots depict the median and ensemble spread at $75 \%$ (box), $90 \%$ (smaller box), and maximum/minimum (whisker lines). The red line depicts the Truth simulation. The black line depicts the JTWC best-track data.

represent the high wind speeds and track location. Therefore, we can conclude that the differences between the CNTRL and the Truth are sufficient to demonstrate the effect of the assimilation in the SCDA system.

\section{b. DA ensemble analysis}

The COAWST-DART system reduces the RMSD in the analysis of the ocean currents by around $30 \%-50 \%$. This is 


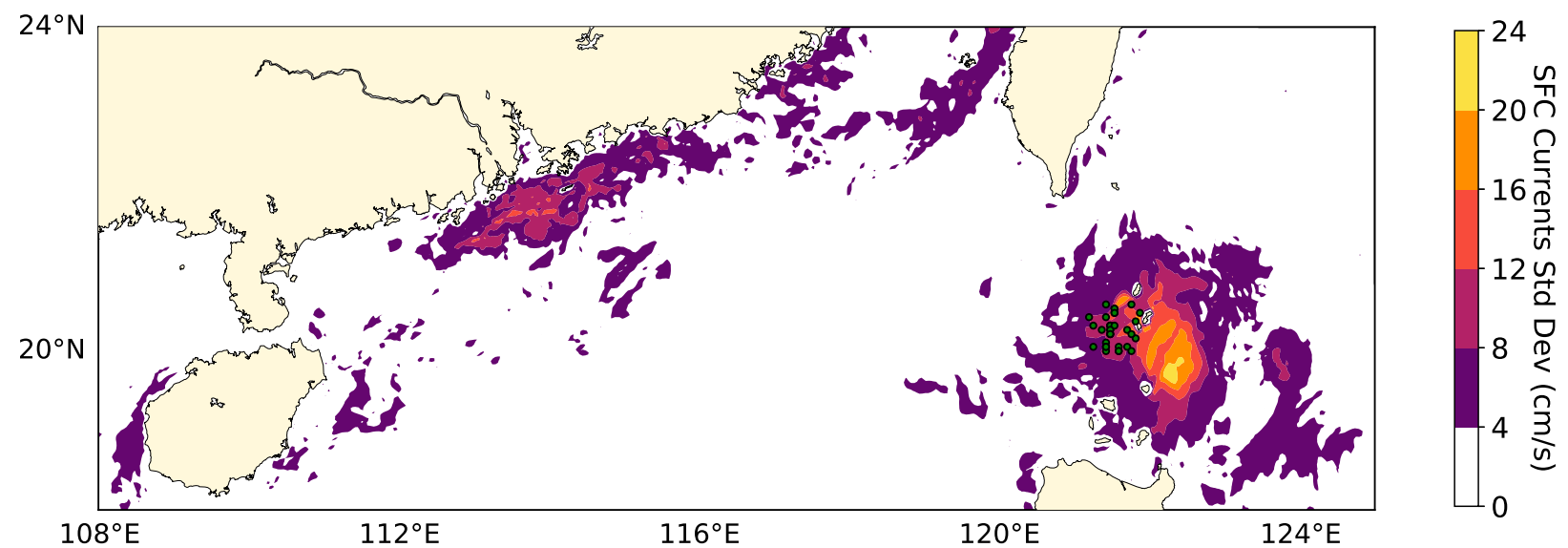

a)

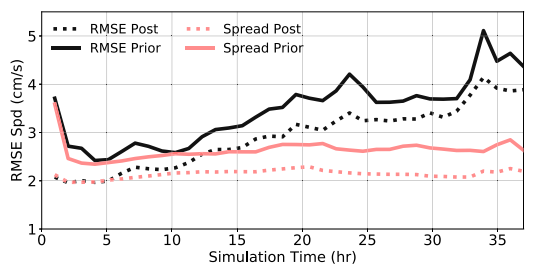

b)

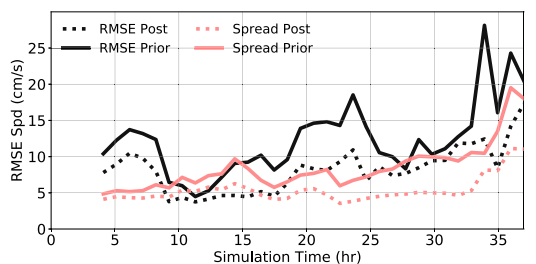

c)

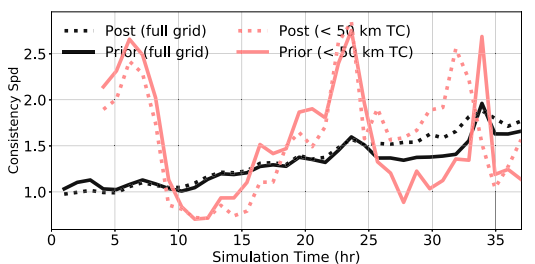

d)

FIG. 4. (a) The standard deviation of the surface current speed $\left(\mathrm{cm} \mathrm{s}^{-1}\right)$ in the initial ensemble at 1800 UTC 21 Aug. Green dots indicate the positions of the TC within the ensemble. (b) The time series of the RMSD between the ocean current speed for the HF+DRIFT experiment and the Truth currents calculated for all assimilated observations within the entire domain (black lines). The prior and posterior RMSD are displayed as solid and dashed lines, respectively. The ensemble spread of HF+DRIFT surface currents is also displayed in red on the same axis. (c) As in (b), but calculated only for observations within $50 \mathrm{~km}$ of the Truth TC center. (d) The ratio of the surface current RMSD and spread (consistency) for the HF+DRIFT experiment. The prior and posterior consistencies are displayed as solid and dashed lines, respectively. The black lines represent the consistency calculated for all observations within the full grid and red lines for within $50 \mathrm{~km}$ of the Truth TC center.

highlighted by the time series of the current speed RMSD between the HF+DRIFT experiment and Truth simulation (Figs. 4b,c). The analysis is split to include all observations in the domain (Fig. 4b) and observations only within $50 \mathrm{~km}$ of the Truth TC (Fig. 4c). The posterior ensemble RMSD (dotted line in Figs. 4b,c) is consistently lower than the prior (solid line in Figs. $4 b, c)$ for both analyses for each hourly cycle.

Within an ensemble DA system, consistency is achieved when the RMSD is equal to the ensemble spread (Wilks 2011), i.e., the ratio of the RMSD and spread is equal to 1 . Variation from this equality results in an ensemble that is either under or overdispersed. When computed for all observations in the domain, the HF+DRIFT ensembles are generally under dispersed (black lines in Fig. 4d), which suggests the system is underfitting most of the observations. The TC acts as the primary driver of spread for the ocean currents, hence isolating the performance of the DA system locally. For example, in the initial ensemble, the majority of the spread is located near the initial cluster of TCs (Fig. 4a). Nevertheless, despite a larger spread, the localized consistency (computed for observations within $50 \mathrm{~km}$ of the Truth TC) exhibits large variability, mostly dominated by underdispersion and only occasionally reduces to one (red lines in Fig. 4d).

We next explore typical cross-domain correlation structures within the $\mathrm{HF}+\mathrm{DRIFT}$ prior ensemble at peak intensity.
These correlations is used to form the sample error covariance $\sigma\left(x_{m, i, j, z}, y\right)$. Prior cross-domain correlations will reveal what unassimilated variables could be impacted (i.e., 3D atmosphere) during the assimilation cycle. Covariances are not examined because variables with large variances dominate prior sample covariances of the coupled state vector. For a fixed location of a single point in the ocean (black dot in Figs. 5a,d,g) the correlations between the ROMS zonal ocean surface current $U$ and full 3D WRF $U$ winds, $V$ winds, and 2D surface pressure (PSFC) are examined over the entire horizontal and vertical $i, j, z$ domain. This correlation is multiplied by the same Gaspari-Cohn localization function as used within COAWSTDART (dashed lines in Figs. 5a,c,d,f,g) with horizontal and vertical half-widths of 150 and $20 \mathrm{~km}$, respectively. The linear regression of the strongest correlation within the domain is also displayed for each WRF variable (Figs. 5b,e,h).

While we only examine the correlation of the surface current with three WRF variables, cross-domain correlations with other atmospheric variables (3D temperature, geopotential height, and mixing ratios of water vapor, rainwater, cloud water, graupel, ice, and snow) are present and adjusted in COAWST-DART during assimilation.

The strongest correlation for the ROMS zonal $(U)$ surface current in the HF+DRIFT prior ensemble is with the WRF U 


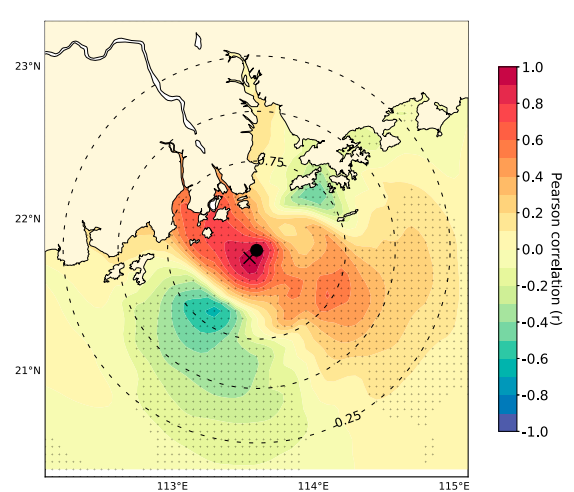

a)

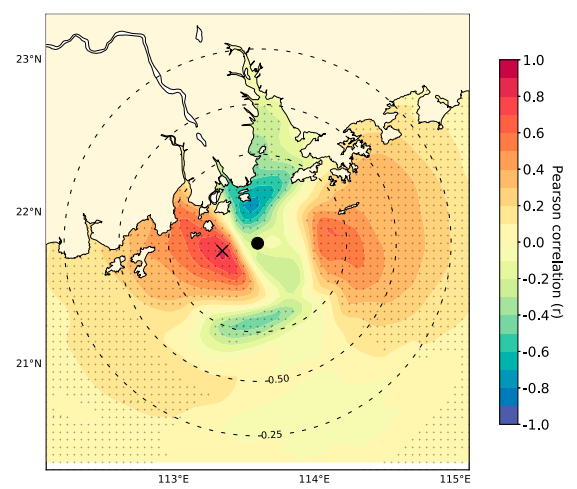

d)

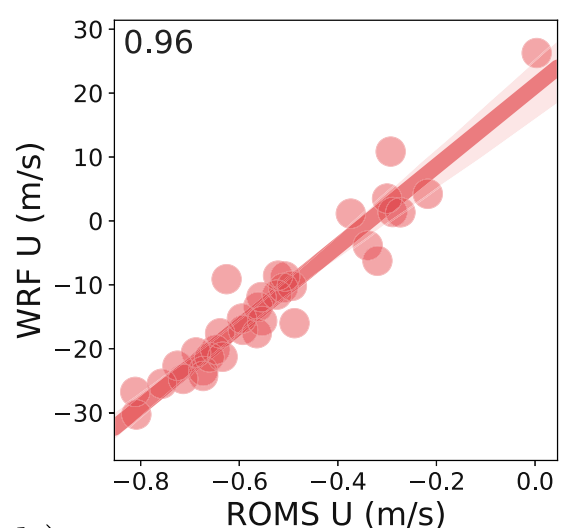

b)

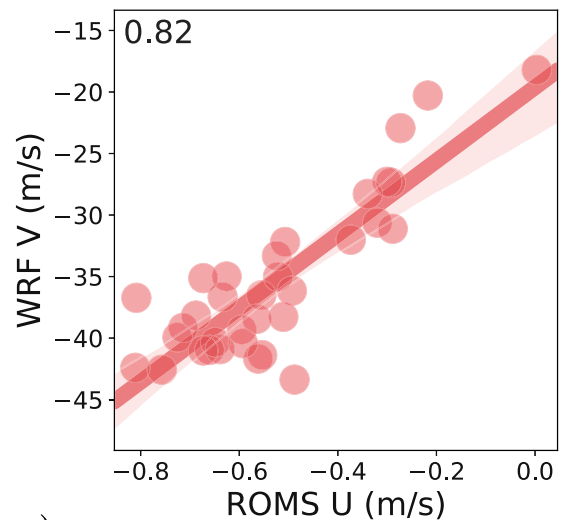

e)

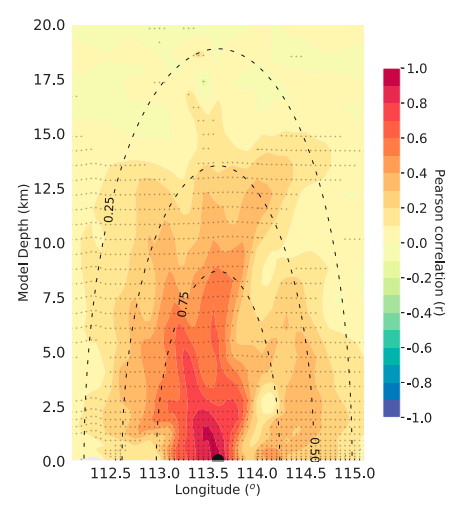

c)

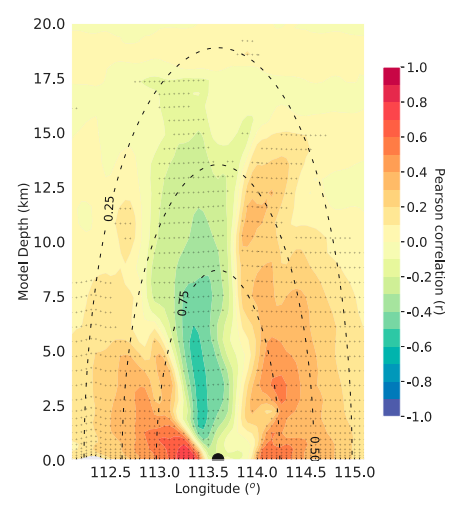

f)

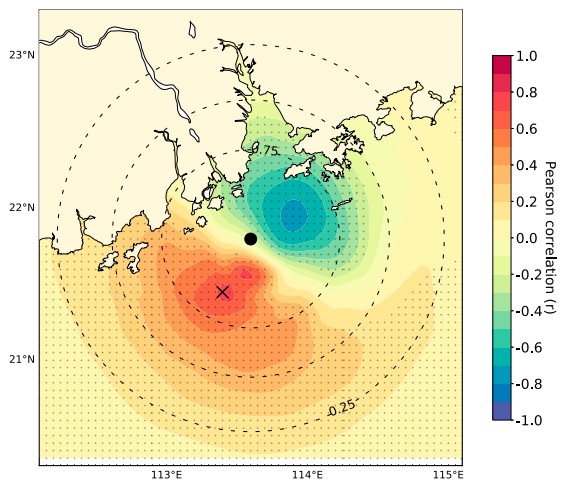

g)

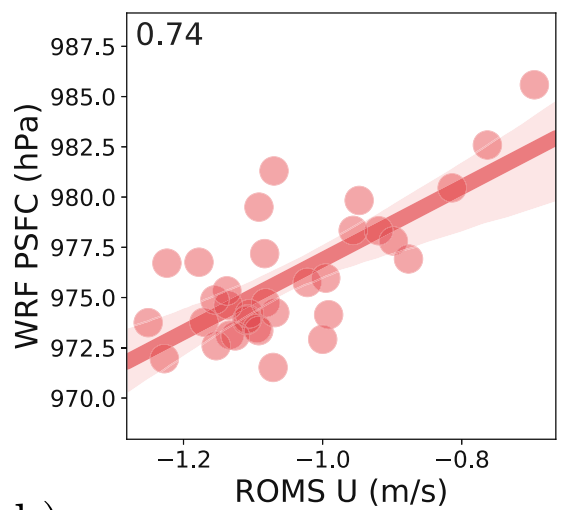

h)

FIG. 5. The correlation of the HF+DRIFT prior ensemble ROMS zonal surface current $U$ at a single fixed point in the ocean (black dot) and the full 3D WRF (top) $U$ and (middle) $V$ winds and (bottom) 2D surface pressure (PSFC) examined over the (a), (d), (g) horizontal and (c),(f) vertical domains. The $\times$ symbol represents the location of the strongest correlation within the spatial pattern. Also shown is (b),(e),(h) the associated linear regression between the ROMS zonal surface current (black dot) and variables located at the $\times$ symbols. The dashed lines in the spatial correlation field represent the localization function applied to the global correlations from the single fixed point.

surface winds (Figs. 5a-c), with a maximum Pearson correlation of 0.95 . This strong positive correlation pattern $(>0.8)$ is centered within $100 \mathrm{~km}$ of the fixed ROMS U location. A weaker negative correlation is also located to the southwest, creating a dipole structure in the correlation field. This dipole structure suggests both an enhancement in the cyclonic circulation and shifting of the TC vortex to the southwest if the assimilation at the fixed point enhances the negative zonal current $U$. The strong positive correlation at the surface vertically extends up to $15 \mathrm{~km}$ (Fig. 5c). This demonstrates the deep vertical structure of Typhoon Hato at peak intensity, indicating our choice of a deep vertical localization is 


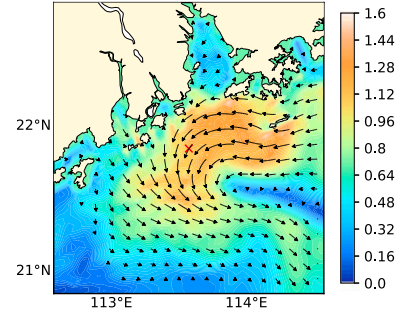

a)

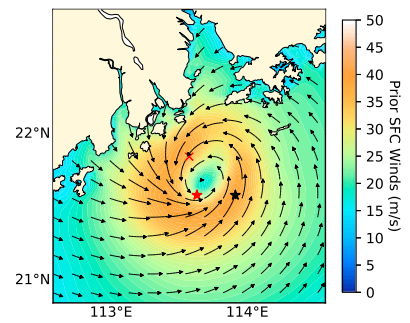

e)

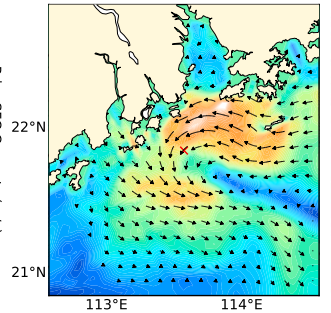

b)

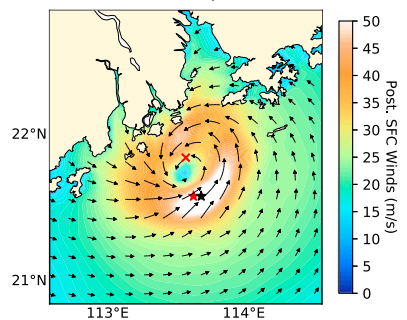

f)

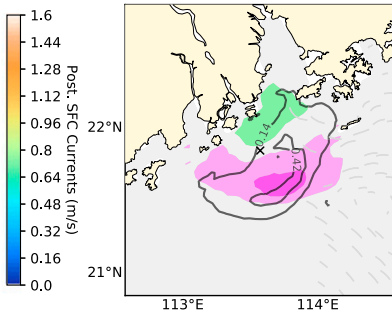

c)

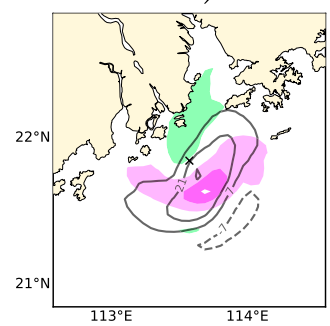

g)

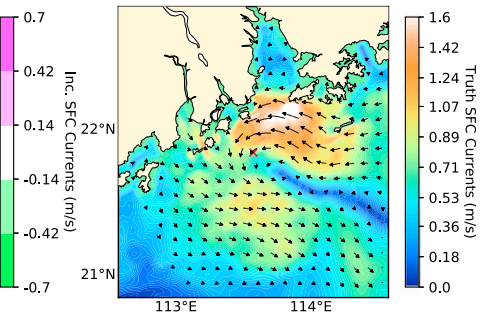

d)

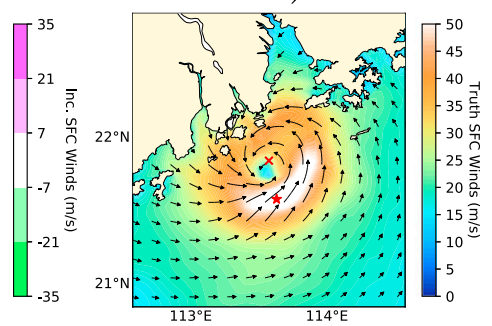

h)

FIG. 6. The HF+DRIFT ensemble mean (a),(e) prior; (b),(f) posterior; (c),(g) increment; and (d),(h) Truth simulation at the peak intensity assimilation cycle 0300 UTC 23 Aug for the ensemble mean (top) ROMS surface current speed and velocity vectors and (bottom) WRF 10-m wind speed and velocity vectors. The red $\times$ depicts the position of the vortex within the nature run to which each frame is centered spanning $\pm 100 \mathrm{~km}$. Increments are split into the $U$ components (shaded color contours) and $V$ components (line contours, with dashed lines representing negative values and solid lines representing positive values). Overlaid in the increments (gray) is the coverage of assimilated data. The black and red stars depict the location of the maximum wind speed in the HF-DRIFT prior and posterior ensemble (Truth simulation), respectively.

reasonable. For the correlation of the ROMS $U$ surface current with the WRF $V$ wind (Figs. $5 \mathrm{~d}-\mathrm{f}$ ), a dipole pattern is also present. This suggests a cyclonic circulation/TC shift similar to that with the correlation with WRF $U$ winds. The correlation of ROMS $U$ surface current with WRF surface pressure (PSFC) resembles a distinct dipole. An enhancement of negative zonal current $U$ at the fixed point will hence produce a deepening of the pressure to the south west shifting the vortex. This is dynamically balanced with the WRF $U$ and $V$ wind correlation structures.

We next explore the direct impact of assimilating ocean currents on the WRF Model for the HF+DRIFT experiment at the peak intensity cycle (0300 UTC 23 August) (Fig. 6). During this cycle, the assimilation of the ocean current dataset produces a distinctive positive zonal current increment (Fig. 6c). This increment, in turn, weakens the westward zonal current in the prior (Fig. 6a), shifting the entire cyclonic circulation slightly westward in the posterior (Fig. 6b). Inertial oscillations are present in the HF radar dataset and could be contributing to the formation of the positive increment. After the assimilation, the posterior mean (Fig. 6b) agrees more with the Truth simulation currents (Fig. 6d) than the prior (Fig. 6a). This ocean current increment is regressed onto the wind vectors [via Eq. (1)] constructing the wind increment (Fig. 6g), similar in shape to the ocean increment (Fig. 6c). This both intensifies TCs within the ensemble and improves the spatial distribution of the wind field. The posterior mean of winds (Fig. 6f) is now remarkably similar to that of the Truth simulation (Fig. 6h). Without the strongly coupled DA system, no such increments are produced in the wind field (not shown).
The assimilation of either the hypothetical HF radar or drifter observed surface currents is also able to produce marked improvements in the TC metrics (track, Pmin, Vmax, R18, and IPD) as compared with the CNTRL and WC experiments (Fig. 7). Comparing the HF, DRIFT and combined HF+DRIFT experiment specifically, the latter combination reduces the average error the most for the majority of TC metrics (Figs. 7a,c,e,g,i). As compared with the CNTRL, the $\mathrm{HF}+\mathrm{DRIFT}$ experiment exhibits a time-average absolute mean ensemble error reduction of $78 \%$ in track $(75 \mathrm{~km}), 57 \%$ in Pmin $(3.8 \mathrm{hPa}), 41 \%$ in $\mathrm{Vmax}(5 \mathrm{kt}), 54 \%$ in $\mathrm{R} 18(17 \mathrm{~km})$, and $70 \%$ in IPD $(0.4 \mathrm{TW})$.

In general, the HF radar dataset provides substantial improvements in all TC metrics over the CNTRL near the coastline. The drifter dataset provides improvements in the Pmin and Vmax over the CNTRL farther afield. This improvement is evident in the time series evolution of each experiment (Fig. 8). In the mean ensemble Pmin and Vmax error (Figs. 8a,c), the DRIFT experiment exhibits a much earlier improved estimate over the CNTRL as compared to HF experiment $(10 \mathrm{~h}$ into the simulation, i.e., $22 \mathrm{~h}$ before the landfall). This is not surprising as the TCs in the ensemble are impacted by the drifters first, and the spatial extent of the drifters is larger than that of the HF radar (Fig. 2). The HF experiment only improves over the CNTRL for Pmin and Vmax after $24 \mathrm{~h}$ when the TC crosses into the vicinity of the HF radar (dashed vertical line in Fig. 8). After a small adjustment, the HF experiment ultimately improves over the DRIFT experiment. 

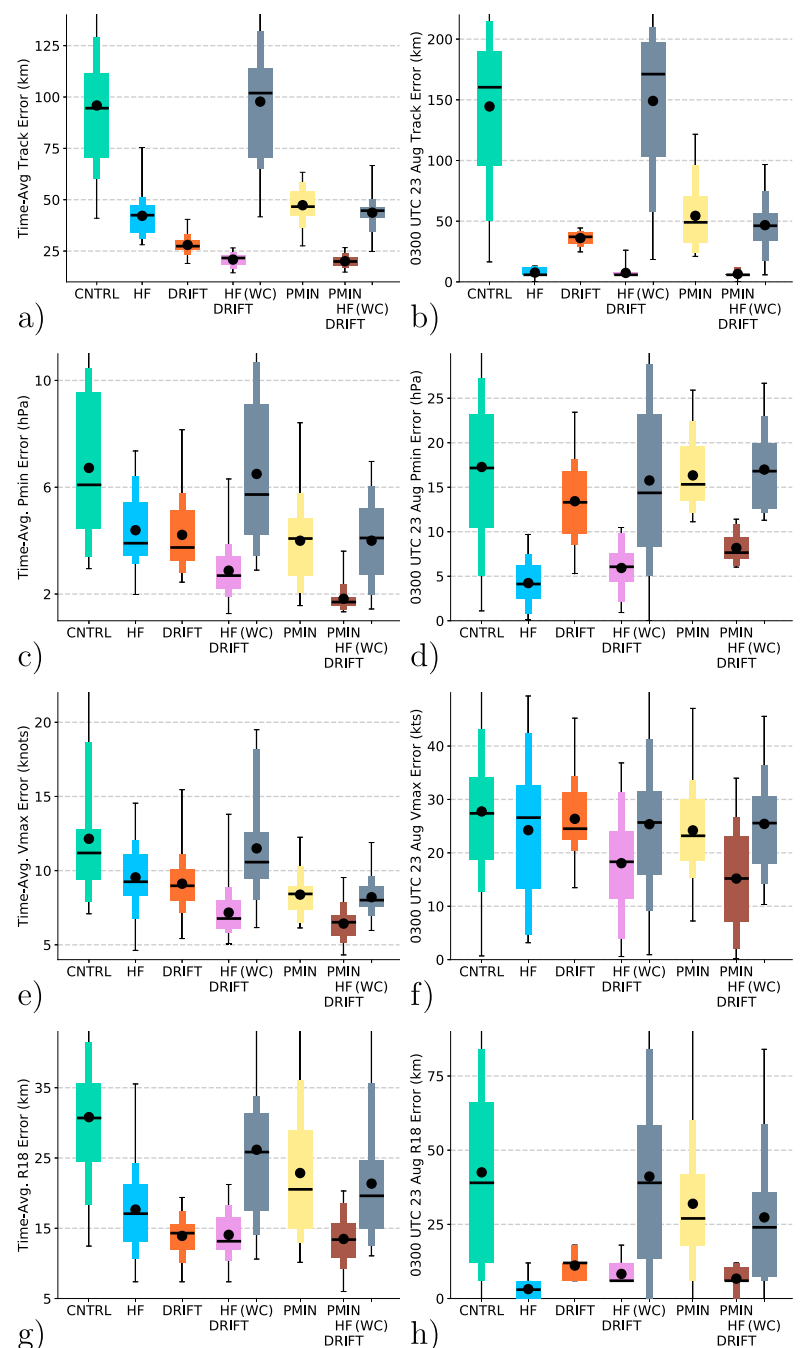

g)
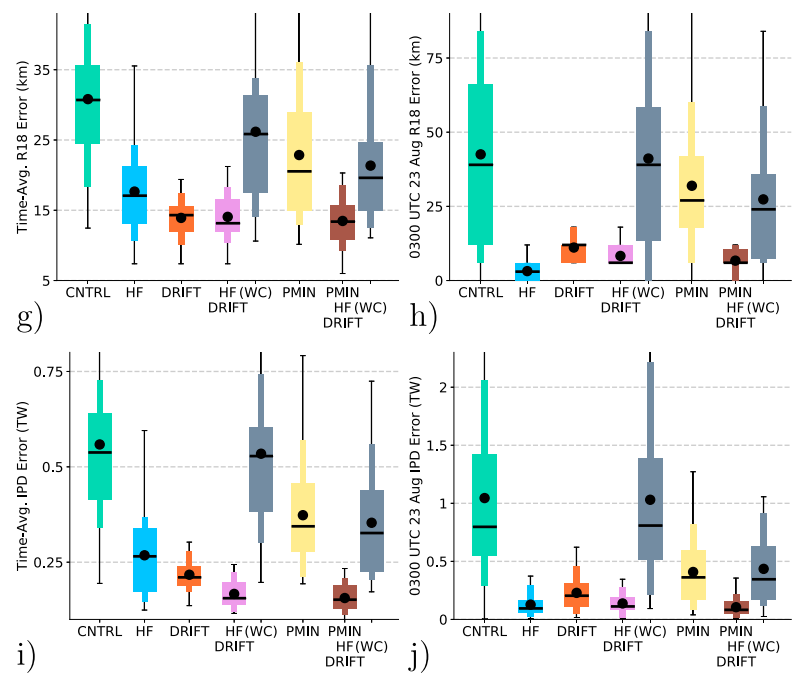

FIG. 7. Box-and-whisker plots depicting each experiment's median (black solid line), mean (black circle), and distribution of (left) time-averaged and (right) peak intensity (0300 UTC 23 Aug) ensemble TC metric errors. The larger box edges represent the error distribution at $75 \%$, small boxes represent the error distribution at $90 \%$, and whisker lines represent the error distribution extending to the maximum/minimum errors. TC metrics include the (a),(b) track; (c),(d) Pmin; (e),(f) Vmax; (g),(h) R18; and (i),(j) IPD. Each experiment is highlighted by the following colors; CNTRL (green), HF (blue), DRIFT (red), HF+DRIFT (pink), PMIN (yellow), and PMIN+HF+DRIFT (brown). Both $\mathrm{HF}+\mathrm{DRIFT} \mathrm{WC}$ and PMIN + HF + DRIFT WC are denoted by (WC) next to their strongly coupled counterparts highlighted in gray.
Interestingly, for the size (R18), the DRIFT experiment exhibits an average improvement over the CNTRL on par with the HF+DRIFT experiment (55\%) (Fig. 7d). However, despite having the lowest R18 error reduction on average, the DRIFT experiment overestimates the R18 between 5 and $24 \mathrm{~h}$ by up to $20 \mathrm{~km}$ (Fig. $8 \mathrm{c}$ ).

For HF+DRIFT, a smooth transition from the drifter's influence to the HF radar's influence is achieved. However, for some metrics (Vmax, Pmin Figs. 8a,c) this combination can be detrimental over the individual assimilation of the HF radar. At peak intensity, the largest reduction in error for almost all TC metrics is found in the HF experiment (Figs. 7b,d,f,h,j). The $\mathrm{HF}$ experiment has an error reduction of $95 \%$ in track $(136 \mathrm{~km}), 75 \%$ in Pmin (13 hPa), 93\% in R18 (40 km), and 88\% IPD (0.9 TW) relative to CNTRL. The assimilation of the HF radar dataset can constrain the overall scale $(100 \mathrm{~km})$ of the TC within the Truth simulation remarkably well with just a 4-km error in R18.

Despite such improvements for most TC metrics at peak intensity, a substantial overestimation of Vmax (up to $20 \mathrm{kt}$ ) is present for all experiments assimilating the HF dataset (Figs. 7d and 8c,d). This can be understood in terms of the cross-domain correlation of wind speeds with the ocean currents. While the relationship between surface winds and ocean currents for most wind speeds is approximately linear, this is not the case for the strongest winds speeds $\left(>35 \mathrm{~m} \mathrm{~s}^{-1}\right)$. For these stronger winds, the drag coefficient within WRF (Green and Zhang 2013) approaches a limiting value, as seen in observations (Donelan et al. 2004). Therefore WRF prior ensemble members that contain TCs with large wind speeds are likely to overestimate the wind stress-ocean current exchange from linear regression, resulting in spurious adjustments.

The PMIN experiment demonstrates that the assimilation of a single atmospheric point can improve the track and intensity estimation (Figs. 7 and 8). As compared with the CNTRL, the PMIN experiment exhibits an average mean ensemble error reduction of $51 \%$ in track $(49 \mathrm{~km}), 41 \%$ in Pmin $(2.7 \mathrm{hPa}), 31 \%$ in $\mathrm{V} \max (3.8 \mathrm{kt}), 26 \%$ in $\mathrm{R} 18(8 \mathrm{~km})$, and $33 \%$ in IPD (0.2 TW). However, at peak intensity, the PMIN experiment underestimates the intensity on par with the CNTRL (Figs. 7d,f and 8a-d). This is due to the construction of the interpolated hourly minimum pressure dataset. At peak intensity, the PMIN experiment is assimilating a weaker minimum pressure than that of the Truth TC. The coarse temporal sampling (every $6 \mathrm{~h}$ ) of the hypothetical "TC guidance product" misses the peak. This sampling error is assimilated into the PMIN experiment.

In combining with the ocean observations (PMIN $+\mathrm{HF}+$ DRIFT), a complementary response is exhibited within the SCDA system for the majority of metrics. As compared with the CNTRL, the PMIN+HF+DRIFT experiment exhibits an average ensemble mean error reduction of $79 \%$ in track $(76 \mathrm{~km}), 73 \%$ in $P \min (4.9 \mathrm{hPa}), 47 \%$ in $\mathrm{Vmax}(5.7 \mathrm{kt}), 56 \%$ in $\mathrm{R} 18(17 \mathrm{~km})$, and $72 \%$ IPD $(0.4 \mathrm{TW})$. This is an improvement upon the PMIN experiment of 58\%, 54\%, 23\%, 41\%, and 58\% in track, Pmin, Vmax, R18, and IPD, respectively.

The spatial wind field error as compared with the Truth simulation at peak intensity is next evaluated (Fig. 9). On average, the HF (Fig. 9b), HF+DRIFT (Fig. 9d) and 


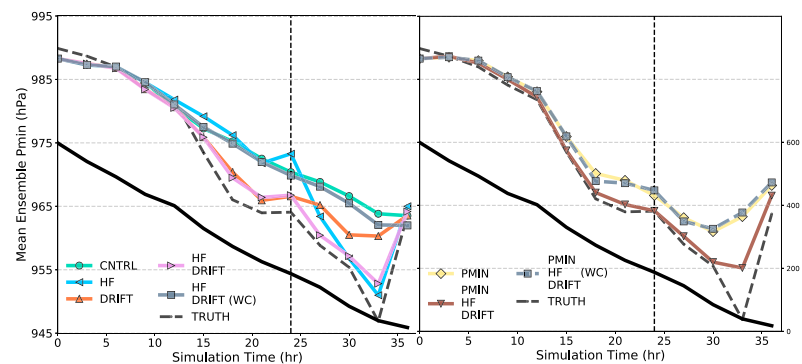

a)

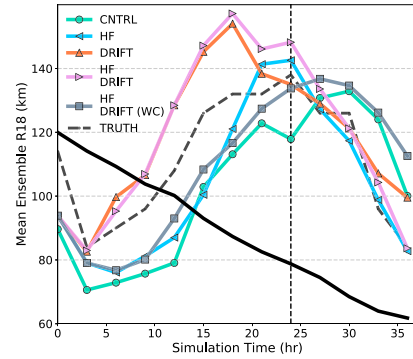

e)

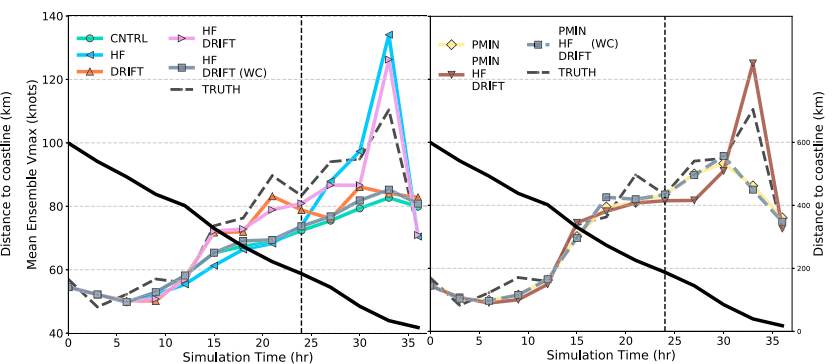

c)

d)

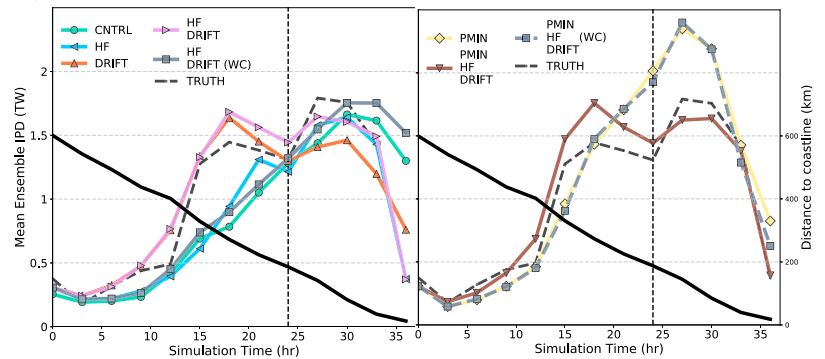

g) h)

FIG. 8. Time series evolution of the mean posterior ensemble (a),(b) Pmin; (c),(d) Vmax; (e),(f) R18; and (g), (h) IPD over the entire 36-h simulation for the CNTRL (solid green line), HF (solid blue line), DRIFT (solid red line), HF+DRIFT (solid pink line), $\mathrm{HF}+\mathrm{DRIFT}$ WC (solid gray line), PMIN (solid yellow line), PMIN+HF+DRIFT (solid brown line), and PMIN + HF + DRIFT WC (dashed gray line). The Truth simulation is depicted with a dashed black line. The right-hand axis in each figure depicts the distance to the coastline $(\mathrm{km})$ and is shown by the solid black line. The vertical dashed line depicts the time at which the Truth TC is directly over the HF radar observations.

PMIN + HF+DRIFT (Fig. 9g) experiments produce the smallest wind field differences. This corresponds to a domainaverage (within $2^{\circ}$ of the Truth TC) RMSD of 2.6, 2.8, and $2.7 \mathrm{~m} \mathrm{~s}^{-1}$. The spatial distribution of the wind field differences (Figs. 9a,c,f) is restricted to the southeastern quadrant where the maximum wind speed in Truth TC is located. Despite difficulties in reproducing the maximum wind speed, the spatial pattern of the Truth wind field is well replicated.

This is contrary to the DRIFT (Fig. 9c), HF+DRIFT WC (Fig. 9e), PMIN (Fig. 9f) and PMIN+HF+DRIFT WC (Fig. 9h) experiments. Here, the spatial extent of the wind field differences envelope the center of the Truth TC (red marker in Fig. 9). This is especially evident for the HF+DRIFT WC experiment with a domain-average RMSD of $12.6 \mathrm{~m} \mathrm{~s}^{-1}$ similar to that of the CNTRL (Fig. 9a). The substantial differences close to the Truth TC center $\left(>21 \mathrm{~m} \mathrm{~s}^{-1}\right)$ indicate that most ensemble members are not correctly located as compared with the Truth. The track error at peak intensity for the $\mathrm{HF}+\mathrm{DRIFT} \mathrm{WC}$ experiment is $150 \mathrm{~km}$ on average.

The impact on the track is displayed in Fig. 10. The HF experiment ensemble (Fig. 10b) initially follows a similar trajectory to the CNTRL (Fig. 10a) but soon rapidly converges upon the arrival of the HF radar coverage (gray area in Fig. 10). In just $6 \mathrm{~h}$, the ensemble mean track errors reduced from 51 to $3 \mathrm{~km}$. For the rapid intensification period from 2100 UTC 22 August to 0300 UTC 23 August, the mean track error is just $4.3 \mathrm{~km}$ averaged over these $6 \mathrm{~h}$. The drifter assimilation similarly constrains the TC vortex in the ensemble (Fig. 10c) except now over almost the entire simulation. The combined experiment of HF+DRIFT (Fig. 10d) further improves upon this performance, reducing the average ensemble-mean track error from $24 \mathrm{~km}$ for the DRIFT experiment ( $33 \mathrm{~km}$ for HF) to $18 \mathrm{~km}$, a $75 \%$ improvement over the CNTRL. For the PMIN experiment, the assimilation of minimum pressure (Fig. 10f), can constrain the ensemble reasonably well, improving the ensemble mean track error by $52 \%$ over the CNTRL. Combining this with the ocean observations for the PMIN+HF+DRIFT experiment (Fig. 10g) improves the track simulation on par with the HF+DRIFT experiment (Fig. 10d). The accuracy of the HF+DRIFT experiment is already superior to PMIN and therefore, does not provide additional large improvements.

Besides the impact on the TC estimate, the ocean state is also impacted by the assimilation in the COAWST-DART system (Fig. 11). The time-averaged and peak intensity spatial SSH, SST and SSS RMSD reveals all experiments improve over the CNTRL. The RMSD is computed only within $100 \mathrm{~km}$ of the Truth TC center to expose the ocean state influential to (or impacted by) the TCs within each ensemble. For the time-averaged SSH RMSD (Fig. 11a), the HF+DRIFT and $\mathrm{PMIN}+\mathrm{HF}+\mathrm{DRIFT}$ experiments exhibit the largest reduction over the CNTRL (64\%). The correct location of TCs within the ensemble could be driving SSH improvements, via correctly capturing TC upwelling processes (Price 1981; Sanford et al. 1987) as also found in (Chen and Zhang 2019). This could also explain the reduction of the SSH RMSD within the PMIN experiment $(28 \%)$. Note at peak intensity (Fig. 11d) the HF experiment reduces the SSH RMSD the most by $77 \%$ over the CNTRL. This is similar to TC metrics, where the assimilation 


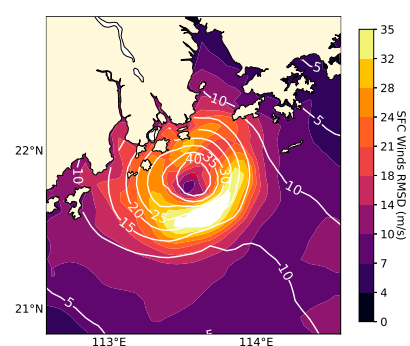

a)

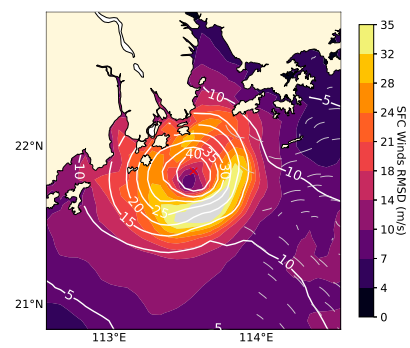

e)

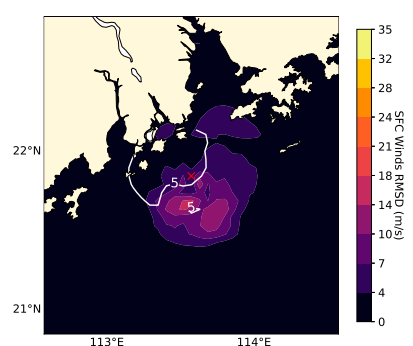

b)

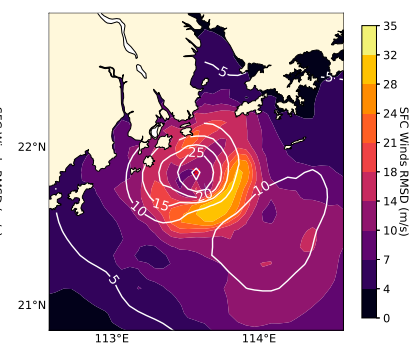

f)

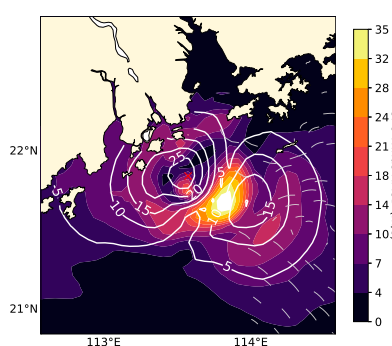

c)

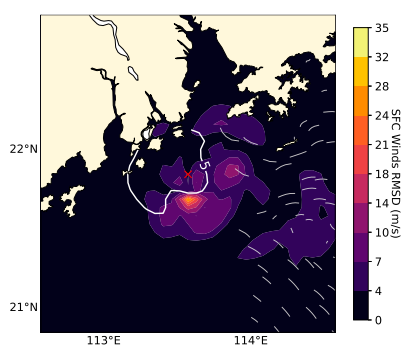

g)

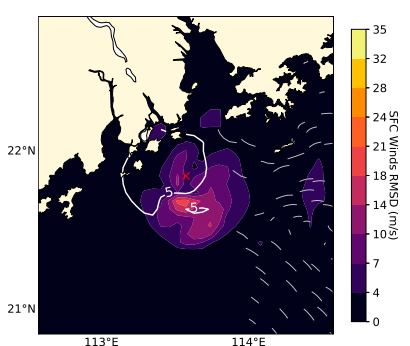

d)

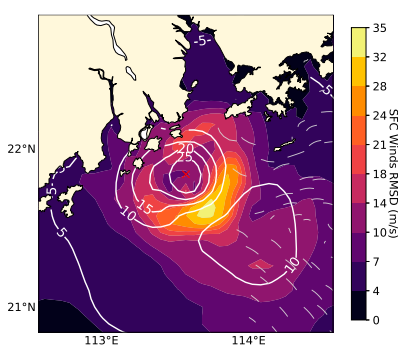

h)

FIG. 9. The average spatial wind errors (shaded contours) at peak intensity (0300 UTC 23 Aug) for the posterior ensemble of the (a) CNTRL, (b) HF, (c) DRIFT, (d) HF+DRIFT, (e) HF+DRIFT WC, (f) PMIN, (g) PMIN+HF+DRIFT, and (h) PMIN+HF+DRIFT WC experiments as compared with the Truth simulation. Overlaid as a white contour is the average spatial pressure errors. The red $\times$ depicts the position of the vortex within the nature run to which each frame is centered spanning $\pm 100 \mathrm{~km}$.

of the HF radar near the coastline strongly constrains the system. For the time-averaged SST RMSD (Fig. 11b), each experiment exhibits a similar improvement over the CNTRL as the SSH RMSD. Here, the HF+DRIFT and PMIN + HF+ DRIFT experiments reduce the SST RMSD by $70 \%$. Finally, for the time-averaged SSS RMSD (Fig. 11c), the experiments with the largest reduction as compared to the CNTRL are those assimilating the HF radar network (71\%).

The first weakly coupled experiment, HF+DIRFT WC (gray solid and labeled in Figs. 7, 8, 10, 11) still produces small average intensity improvements upon the CNTRL (3\%-10\%) but cannot provide the degree of improvement as in the strongly coupled variant $(41 \%-57 \%)$. This is because the crossdomain increments directly impacting the atmospheric variables are not implemented, with the TCs within the ensemble left unconstrained. Instead, only variables within ROMS are adjusted (such as the SST, SSH and salinity). The track therefore (Fig. 10d) evolves similarly to the CNTRL (Fig. 3d), resulting in an error of up to $150 \mathrm{~km}$. Furthermore, as a result of the large spread between ensemble members, the spatial wind field error at peak intensity is also substantially large (Fig. 9d) with a domain-average mean ensemble RMSD of $12.6 \mathrm{~m} \mathrm{~s}^{-1}$ (Fig. 9d). However, the HF+DIRFT WC experiment can improve the ocean state significantly over the CNTRL. For example, the SSH RMSD of HF+DIRFT WC exhibits a reduction of $47 \%$. This is also in agreement with Carrier et al. (2016) and Phillipson and Toumi (2017) who showed that the assimilation of ocean currents could constrain the SSH.

The second weakly coupled experiment, $\mathrm{PMIN}+\mathrm{HF}+\mathrm{DIRFT}$ WC (gray dashed and labeled in Figs. 7, 8, 10, and 11) evolves almost identically as to the PMIN experiment indicating the ocean observations cannot did not constrain the atmospheric variables. Instead only the PMIN dataset impacts atmospheric variables as also in the PMIN experiment. However, marginal improvements upon the PMIN experiment for the intensity and track are evident $(2 \%-8 \%)$. An improved ocean state (Fig. 11) is likely to have provided a small benefit to the coupled TC system as in Dong et al. (2017). Nevertheless, this improvement is significantly smaller as compared with the improvement exhibited by the strongly coupled counterpart PMIN + HF+DRIFT (23\%-58\%).

\section{c. Forecasts}

Figure 12 shows the evolution of the PMIN, PMIN+HF+ DRIFT, and PMIN + HF+DRIFT WC forecasts. For the Pmin predictions, all five $\mathrm{PMIN}+\mathrm{HF}+\mathrm{DRIFT}$ forecasts are closer to the Truth TC Pmin at peak intensity, improving over the PMIN forecasts by $4 \%-32 \%(1-5 \mathrm{hPa})$ and over the WC forecasts by $6 \%-33 \%(2-7 \mathrm{hPa})$.

For the Vmax predictions, the majority of the PMIN + HF+ DRIFT forecasts (0600 UTC 22 August, 1200 UTC 22 August, 1800 UTC 22 August, 0000 UTC 23 August) are also closer to the Truth TC than the PMIN and WC forecasts by $5 \%-31 \%$ $(1-7 \mathrm{kt})$. For the 0000 UTC 22 August forecast, the Vmax is slightly less accurate and cannot improve upon the PMIN or WC forecast predictions.

For the R18 and IPD predictions, the performance of the PMIN+HF+DRIFT early forecasts (0000 UTC 22 August, 0600 UTC 22 August, 1200 UTC 22 August) is variable, with the PMIN forecasts often closer to the Truth TC. However, for the 1800 UTC 22 August and 0000 UTC 23 August PMIN+ HF+DRIFT forecasts, the availability of the HF radar vastly improves the prediction of R18 and IPD (up to $76 \%$ ). 


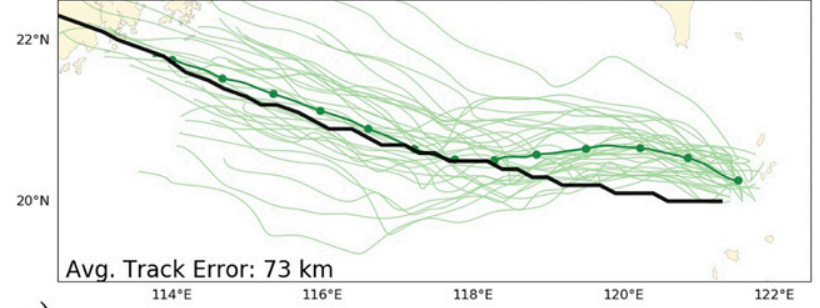

a)

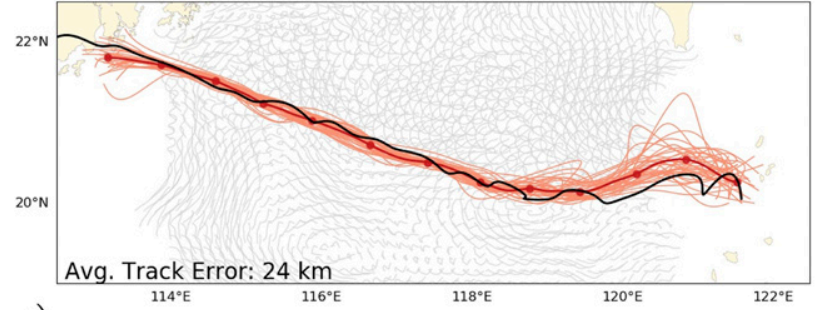

c)

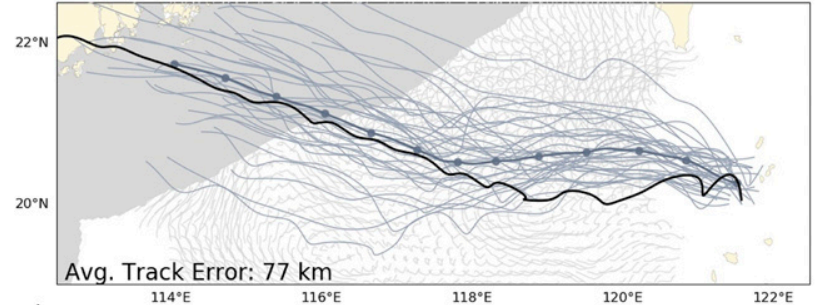

e)

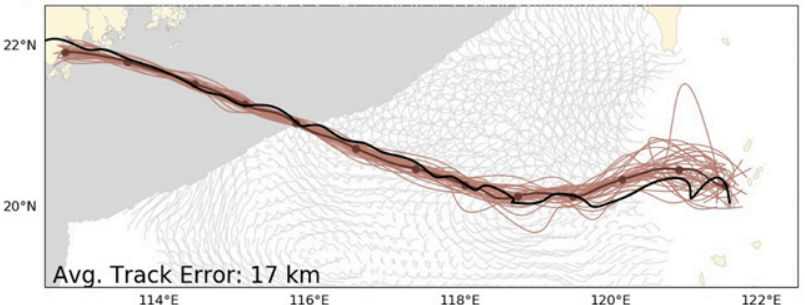

g)
Avg. Track Error: $17 \mathrm{~km}$

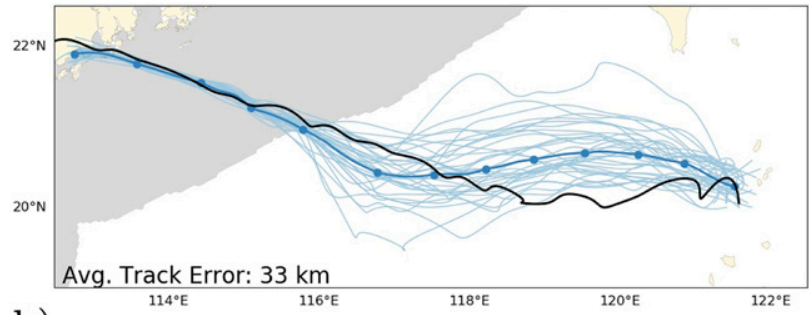

b)

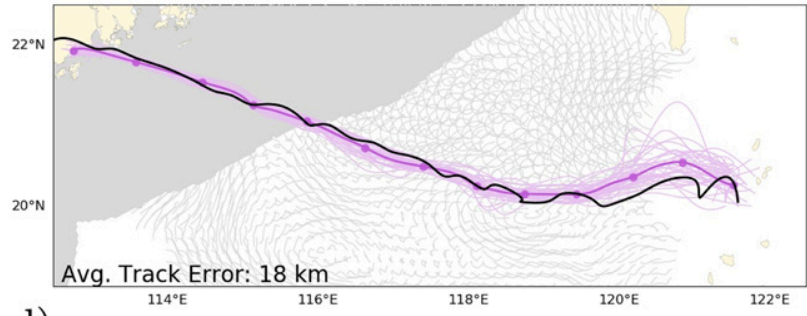

d)

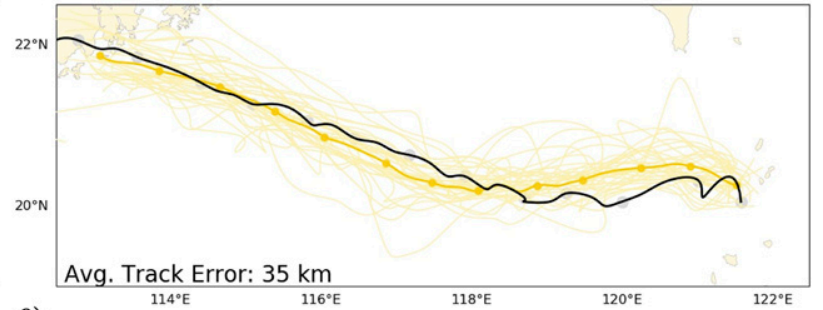

f)

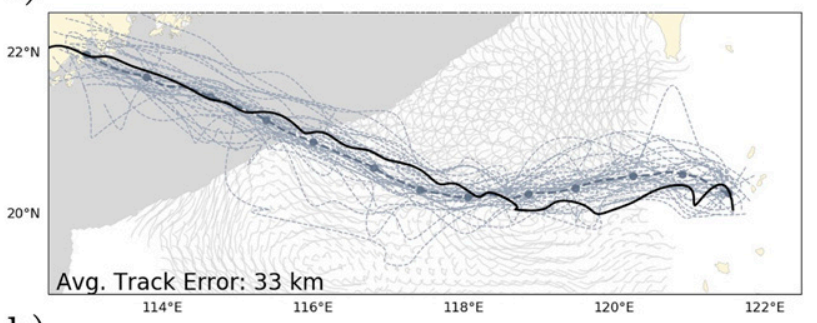

h)

FIG. 10. The TC tracks within the (a) CNTRL, (b) HF, (c) DRIFT, (d) HF+DRIFT, (e) HF+DRIFT WC, (f) PMIN, (g) PMIN+HF+DRIFT, and (h) PMIN+HF+DRIFT WC posterior ensemble. The Truth simulation is overlaid as a black solid line.

For the track predictions, the PMIN + HF+DRIFT forecasts improve the position of the peak intensity as compared to the PMIN and WC forecasts $(15 \%-56 \%)$. However, this is mostly due to the improvements in along-track error rather than the cross-track error, which tends to increase. This results in a southward bias for the location of the peak intensity.

\section{Discussion}

The assimilation of ocean currents observed by drifters (assuming complete coverage in the SCS) and (or) observed by $\mathrm{HF}$ radar (near the coastline with improved resolution) could significantly improve estimates of Typhoon Hato. This result is complementary to both $\mathrm{Li}$ and Toumi (2018) and Chen and Zhang (2019) finding improvements in TCs estimates in an SCDA system. Strong cross-domain correlations in the surface ocean currents and the winds above can be fully utilized within the sample covariance to reverse engineer the influence of TC winds on the ocean. This unlocks the broad potential of TC information contained within ocean current observations. The high temporal resolution of ocean current observations (hourly) is particularly useful for capturing Typhoon Hato's rapid intensification near landfall, producing track, intensity, and size improvements.

The track errors are substantially reduced when the HF radar observations become in range of the TC. The drifter assimilation can also exhibit track improvements far from the coastline. This surface ocean current data assimilation could, therefore supplement current satellite-based estimates for rapid and increased vortex position accuracy (Zhao et al. 2020). Despite improvements in the track and intensity, the drifter assimilation tends to overestimate the radius of galeforce wind for the majority of the simulation. This result contradicts our expectation that the large spatial extent of the 


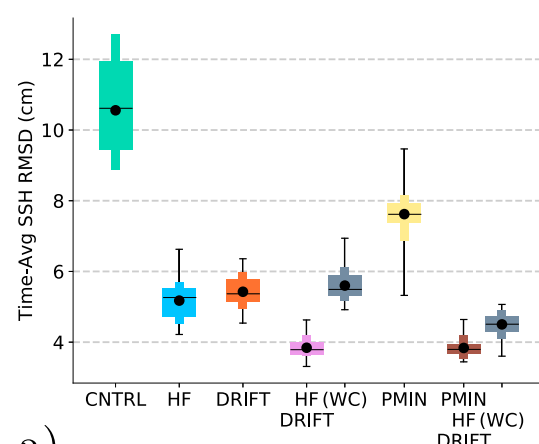

a)

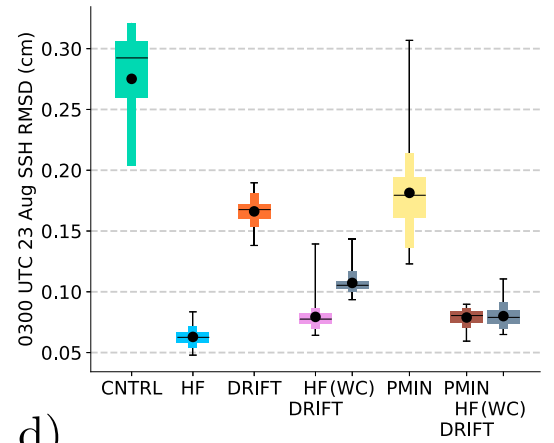

d)

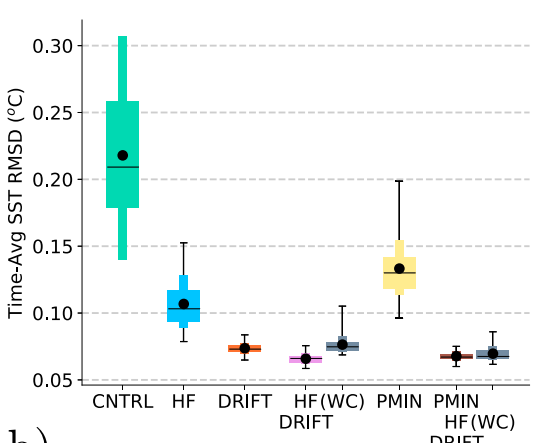

b)
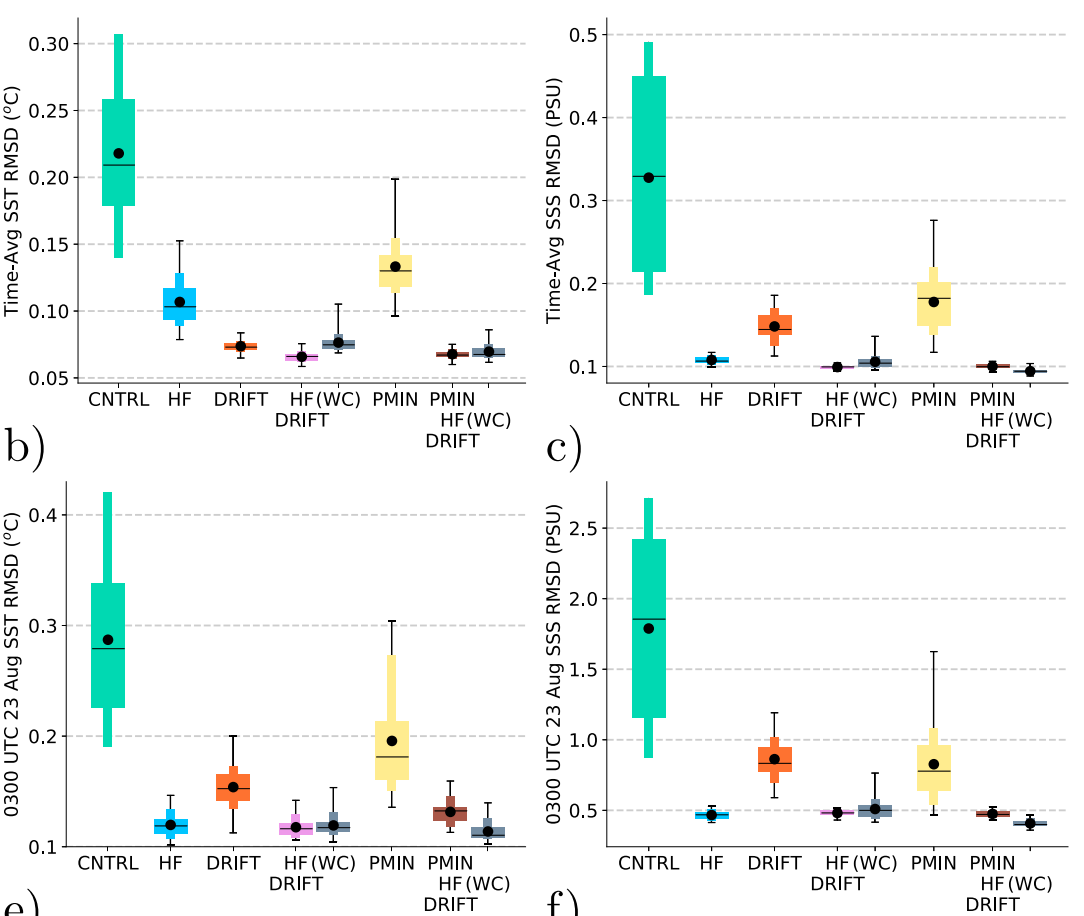

e)

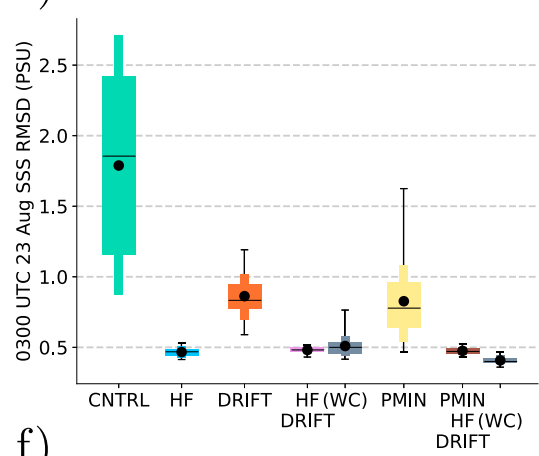

FIG. 11. Box-and-whisker plot depicting each experiment's median (black solid line), mean (black circle), and distribution of (top) timeaveraged and (bottom) peak intensity (0300 UTC 23 Aug) ensemble ocean state RMSD (as compared with the Truth ocean state) computed only within $100 \mathrm{~km}$ of the Truth TC center. Ocean states include the (a),(d) sea surface height; (b),(e) sea surface temperature; and (c),(f) sea surface salinity.

drifter assimilation would be able to constrain information on the outer circulation (Fig. 2). We suspect the effective horizontal resolution of the drifters $(16 \mathrm{~km})$ could be too coarse to adequately constrain the true scale of the TC in the Truth simulation. Nevertheless, in our case of a rapid-moving typhoon, coverage beyond the HF range is beneficial for better track and intensity estimates.

For a real deployment of this scale, challenges remain in postprocessing the drifter location data in real time, as well as successfully collecting the data under hurricane conditions (Goni et al. 2017). The LASER drifter campaign (Haza et al. 2018) has shown that cheap, biodegradable drifters on the order of 1000 are feasible (Novelli et al. 2017). For the LASAR campaign, Novelli et al. (2017) specifically designed a low-cost drifter with $80 \%$ biodegradable material. Using this design, they deployed 1100 units into the Gulf of Mexico. To deploy such an array in the SCS (as proposed in this paper) could cost on the order of USD 1-2 million (NOAA Office of Ocean Exploration and Research 2005). However, we acknowledge that the optimistic coverage of drifters outlined in this study (e.g., the wide, dense, and instantaneous distribution) would be difficult to achieve, and is instead a proof-of-concept. For a future real-time forecast, targeted deployment could aid in dispersing the initial drifters to achieve a similar coverage (Poje et al. 2002). If deployed early, the drifters could be of use for the whole duration of the main TC season (July to September). Furthermore, each TC event would naturally expand the spatial coverage of the drifter array.
The HF radar dataset we envisaged along the entire southern coast of China is technically achievable. An excess of 60 radars would need to be built, costing more than USD 30 million (Anderson 2014). It would have benefits for multiple typhoon forecasts, as well as oil spill tracking and rescue operations. Nevertheless, it is difficult to estimate the exact cost-benefit of an improved intensity analysis and forecast.

A weakly coupled system cannot provide such dramatic improvements in TC metrics as we show for the strongly coupled system. This result is similar both that found in $\mathrm{Li}$ and Toumi (2018) and Chen and Zhang (2019). The instant crossdomain correlation update via the prior sample covariance works remarkably efficiently because the TC winds govern the surface currents. For example, a typical strongly coupled operational DA system would usually suffer from dramatic differences in temporal and spatial scales between ocean and atmosphere. This dissonance would require significant modifications to the prior cross-domain sample covariance via reconditioning and (or) specific tuning of localization techniques [Smith et al. 2018; Z. Shen et al. 2018, unpublished manuscript (https://doi.org/10.5194/npg-2018-50); Penny et al. 2019] or an interface solver (an approximation to the SCDA problem) (Frolov et al. 2016). However, for TCs and ocean current assimilation, the strongly coupled data assimilation problem is simplified. Even when the spread of the ensemble vortex position is minimal $(<10 \mathrm{~km})$, the dramatic difference between wind speeds inside the eyewall $\left(50 \mathrm{~m} \mathrm{~s}^{-1}\right)$ and eye $\left(6-10 \mathrm{~m} \mathrm{~s}^{-1}\right)$ creates sufficient spread in the ocean 
a) $00 \mathrm{Z} / 22$

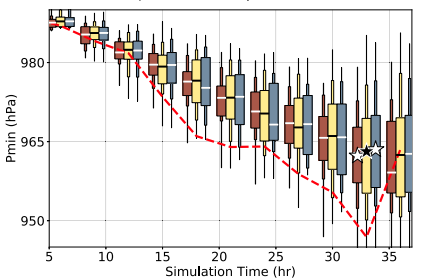

1.a)

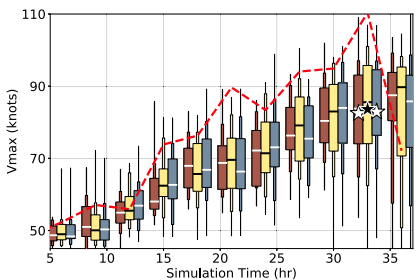

2.a)

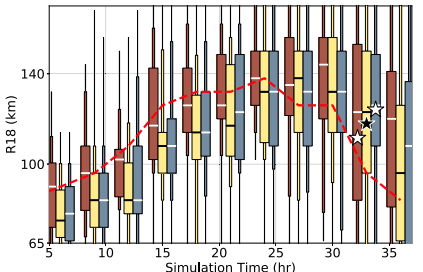

3.a)

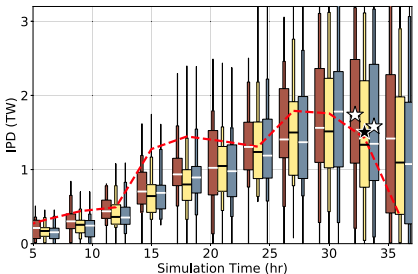

4.a)

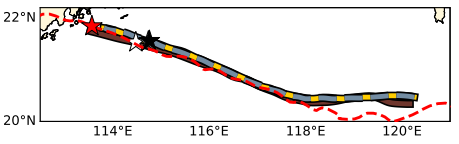

5.a) b) $06 \mathrm{Z} / 22$

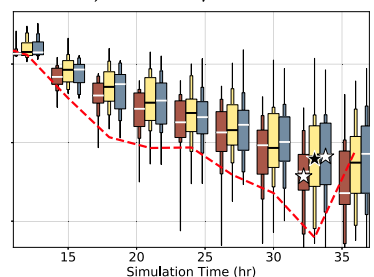

1.b)

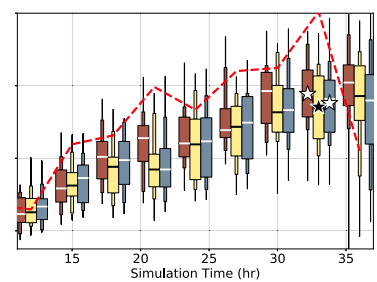

2.b)

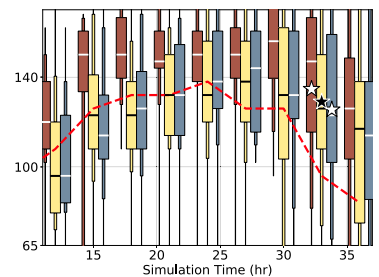

3.b)

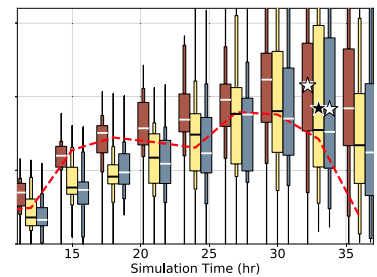

4.b) c) $12 \mathrm{Z} / 22$

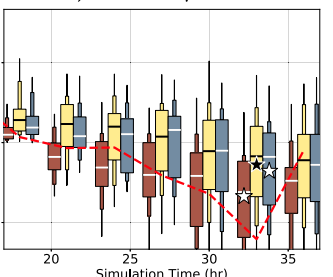

1.c)

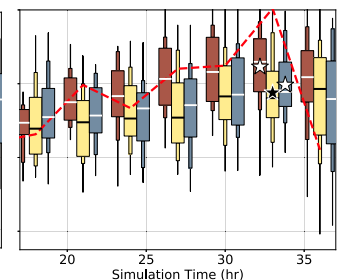

2.c)

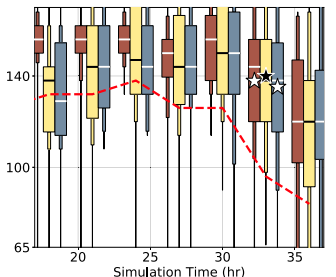

3.c)

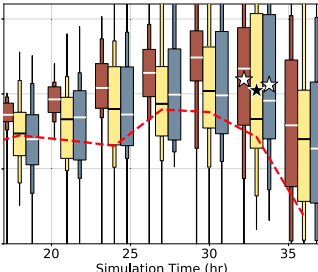

4.c)

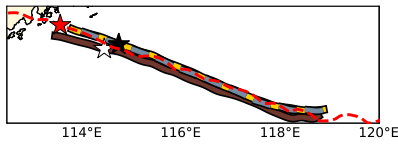

5.b)

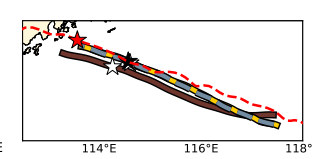

5.c) d) $18 \mathrm{Z} / 22$

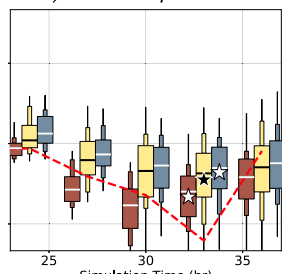

1.d)

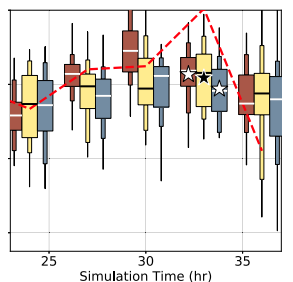

2.d)

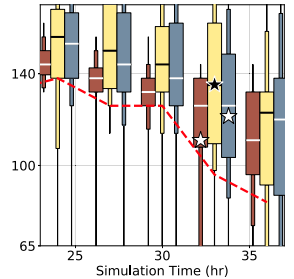

3.d)

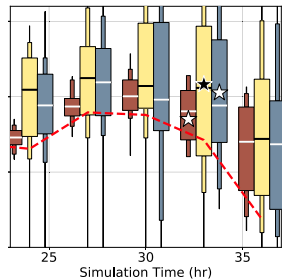

4.d)

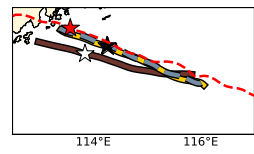

5.d) e) $00 \mathrm{Z} / 23$

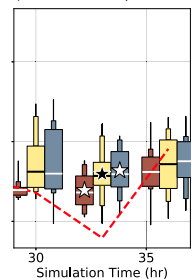

1.e)

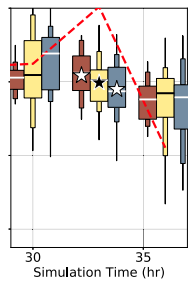

2.e)

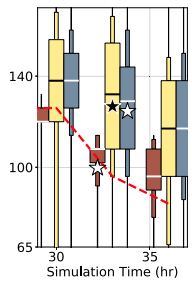

3.e)

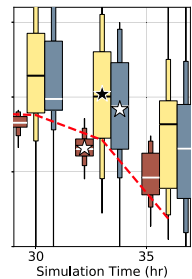

4.e)

FIG. 12. Five ensemble forecasts for the PMIN+HF+DRIFT (brown lines/box), PMIN (yellow lines/box), and PMIN +HF+DRIFT WC (gray dashed lines/box) experiments initialized at (a) 0000 UTC 22 Aug, (b) 0600 UTC 22 Aug, (c) 1200 UTC 22 Aug, (d) 1800 UTC 22 Aug, and (e) 0000 UTC 23 Aug. TC metrics displayed include the (top) Pmin, (top middle) Vmax, (middle) R18, (bottom middle) IPD, and (bottom) tracks. The box-and-whisker plots depict the median and ensemble spread at $75 \%$ (box), 90\% (smaller box), and maximum/minimum (whisker lines). The red dashed line depicts the Truth simulation. The star symbol highlights the mean ensemble TC metric (rows 1-4) and track position (bottom row) at peak intensity (0300 UTC 23 Aug) for each forecast.

surface variables being forced by the winds. The correlation then forms instantaneously, allowing an update to the wind speeds for a particular ocean current observation.

However, for the case of extreme wind speeds and the associated nonlinearity for ocean currents drag (Donelan et al. 2004), we identified the need for an improved regression technique. Future applications could consider nonlinear crosscorrelations updates, such as the rank regression algorithm as described by Anderson (2019). Despite this limitation, the linear correlations are shown to provide an extremely effective way of updating the atmospheric model variables, shifting the TC vortex and enhancing the intensity.

While the results presented in this study are promising, clearly more atmospheric observations (dropsondes, coastal or airborne radar, and atmospheric motion vectors) are assimilated either operationally or experimentally (e.g., Pu et al. 2008, 2009; Weng and Zhang 2012; Dong and Xue 2013). Assimilating a full suite of atmospheric observations is beyond the scope of this study. Nevertheless, we can still discuss possible outcomes. The operational forecast error for 
rapid intensified TCs is $10.8 \mathrm{~m} \mathrm{~s}^{-1}$ (Leroux et al. 2018), similar to the error from the assimilation of TC minimum pressure in this study. When ocean currents are assimilated the error can be further reduced by up to $3.6 \mathrm{~m} \mathrm{~s}^{-1}$, alluding to the possible improvement in an operationally setting. We strongly encourage future investigations of SCDA systems for TC research to employ real ocean current and atmospheric data to test these possibilities.

\section{Conclusions}

The assimilation of ocean currents has been shown to improve estimates of the track, intensity, and overall spatial wind distribution of Typhoon Hato. A combination of (or individually) assimilating hypothetical coastal HF observations, drifter float currents, and minimum surface pressure was examined. Significant benefits (with a reduction of error similar to the order of current operational forecast error) were exhibited in the analysis and subsequent forecasts for TC intensity and track metrics. This is achieved through the prior cross-domain sample covariance via strongly coupled data assimilation. Such a system is especially useful for intense TCs with fast translation speeds. The coastal HF observations and drifter floats provide different benefits to the system. A dense network of drifters could provide coverage farther away from the coast where the HF radar network operates effectively. Such an observation network could be incredibly beneficial for exposed areas with large populations such as the Pearl River Delta.

Acknowledgments. The authors acknowledge funding from the National Key Research and Development Program (2017YFA0604202), the National Natural Science Foundation of China (42006036), Hohai University (B210202141), the U.K.-China Research and Innovation Partnership Fund through the Met Office Climate Science for Service Partnership (CSSP) China as part of the Newton Fund, and the Horizon 2020 EU Research and Innovation program. We thank all of the members of the DART Team at the NCAR Mesa Laboratory and especially Nancy Collins, whose insightful conversations helped to improve the COAWST-DART simulations. John Warner and his COAWST modeling workshop (2019) also provided excellent guidance on the COAWST system. The authors also thank the Imperial College High Performance Computing Service (https://doi.org/10.14469/hpc/2232) for providing computational resources and two anonymous reviewers for providing helpful comments and suggestions on earlier drafts of the paper.

Data availability statement. The numerical model simulations upon which this study is based are too large to archive or to transfer. The model code, compilation script, initial and boundary condition files, and the namelist settings are instead available from the corresponding author $\mathrm{Yi} \mathrm{Li}$ on request.

\section{REFERENCES}

Aksoy, A., S. Lorsolo, T. Vukicevic, K. J. Sellwood, S. D. Aberson, and F. Zhang, 2012: The HWRF Hurricane Ensemble Data Assimilation System (HEDAS) for high-resolution data: The impact of airborne Doppler radar observations in an OSSE.
Mon. Wea. Rev., 140, 1843-1862, https://doi.org/10.1175/ MWR-D-11-00212.1.

Anderson, J. L., 2001: An ensemble adjustment Kalman filter for data assimilation. Mon. Wea. Rev., 129, 2884-2903, https://doi.org/ 10.1175/1520-0493(2001)129<2884:AEAKFF $>2.0 . C O ; 2$.

_ 2003: A local least squares framework for ensemble filtering. Mon. Wea. Rev., 131, 634-642, https://doi.org/10.1175/15200493(2003)131<0634:ALLSFF>2.0.CO;2.

- 2007 : An adaptive covariance inflation error correction algorithm for ensemble filters. Tellus, 59A, 210-224, https:// doi.org/10.1111/j.1600-0870.2006.00216.x.

_ 2009: Spatially and temporally varying adaptive covariance inflation for ensemble filters. Tellus, 61A, 72-83, https:// doi.org/10.1111/j.1600-0870.2008.00361.x.

, 2019: A nonlinear rank regression method for ensemble Kalman filter data assimilation. Mon. Wea. Rev., 147, 28472860, https://doi.org/10.1175/MWR-D-18-0448.1.

—, T. Hoar, K. Raeder, H. Liu, N. Collins, R. Torn, and A. Avellano, 2009: The Data Assimilation Research Testbed: A community facility. Bull. Amer. Meteor. Soc., 90, 1283-1296, https://doi.org/10.1175/2009BAMS2618.1.

Anderson, S. J., 2014: HF radar network design for remote sensing of the South China Sea. Advanced Geoscience Remote Sensing, M. Marghany, Ed., IntechOpen, Rijeka, https:// doi.org/10.5772/57599.

Aydoğdu, A., and Coauthors, 2018: OSSE for a sustainable marine observing network in the Sea of Marmara. Nonlinear Processes Geophys., 25, 537-551, https://doi.org/10.5194/ npg-25-537-2018.

Barker, D. M., W. Huang, Y.-R. Guo, A. J. Bourgeois, and Q. N. Xiao, 2004: A three-dimensional variational data assimilation system for MM5: Implementation and initial results. Mon. Wea. Rev., 132, 897-914, https://doi.org/10.1175/1520-0493(2004) 132<0897:ATVDAS $>2.0$. CO;2.

Bender, M. A., I. Ginis, R. Tuleya, B. Thomas, and T. Marchok, 2007: The operational GFDL coupled hurricane-ocean prediction system and a summary of its performance. Mon. Wea. Rev., 135, 3965-3989, https://doi.org/10.1175/2007MWR2032.1.

Brusdal, K., J. M. Brankart, G. Halberstadt, G. Evensen, P. Brasseur, P. J. van Leeuwen, E. Dombrowsky, and J. Verron, 2003: A demonstration of ensemble-based assimilation methods with a layered OGCM from the perspective of operational ocean forecasting systems. J. Mar. Syst., 40-41, 253-289, https://doi.org/ 10.1016/S0924-7963(03)00021-6.

Cangialosi, J. P., E. Blake, M. DeMaria, A. Penny, A. Latto, E. Rappaport, and V. Tallapragada, 2020: Recent progress in tropical cyclone intensity forecasting at the National Hurricane Center. Wea. Forecasting, 35, 1913-1922, https:// doi.org/10.1175/WAF-D-20-0059.1.

Carrier, M. J., H. Ngodock, S. Smith, G. Jacobs, P. Muscarella, T. Ozgokmen, B. Haus, and B. Lipphardt, 2014: Impact of assimilating ocean velocity observations inferred from Lagrangian drifter data using the NCOM-4DVAR. Mon. Wea. Rev., 142, 1509-1524, https://doi.org/10.1175/MWRD-13-00236.1.

- _ _ P. Muscarella, and S. Smith, 2016: Impact of assimilating surface velocity observations on the model sea surface height using the NCOM-4DVAR. Mon. Wea. Rev., 144, 10511068, https://doi.org/10.1175/MWR-D-14-00285.1.

Chang, Y.-C., R.-S. Tseng, P. C. Chu, J.-M. Chen, and L. R. Centurioni, 2016: Observed strong currents under global tropical cyclones. J. Mar. Syst., 159, 33-40, https://doi.org/ 10.1016/j.jmarsys.2016.03.001. 
Chao, Q., and J. Chao, 2012: Statistical features of tropical cyclones affecting China and its key economic zones. Acta Meteor. Sin., 26, 758-772, https://doi.org/10.1007/s13351-012-0607-0.

Chen, X., and F. Zhang, 2019: Development of a convectionpermitting air-sea-coupled ensemble data assimilation system for tropical cyclone prediction. J. Adv. Model. Earth Syst., 11, 3474-3496, https://doi.org/10.1029/2019MS001795.

Chen, Y., and C. Snyder, 2007: Assimilating vortex position with an ensemble Kalman filter. Mon. Wea. Rev., 135, 1828-1845, https://doi.org/10.1175/MWR3351.1.

Coelho, E. F., and Coauthors, 2015: Ocean current estimation using a multi-model ensemble Kalman filter during the Grand Lagrangian Deployment Experiment (GLAD). Ocean Modell., 87, 86-106, https://doi.org/10.1016/j.ocemod.2014.11.001.

Delandmeter, P., and E. van Sebille, 2019: The Parcels v2.0 Lagrangian framework: New field interpolation schemes. Geosci. Model Dev., 12, 3571-3584, https://doi.org/10.5194/gmd-12-35712019.

DeMaria, M., C. R. Sampson, J. A. Knaff, and K. D. Musgrave, 2014: Is tropical cyclone intensity guidance improving? Bull. Amer. Meteor. Soc., 95, 387-398, https://doi.org/10.1175/ BAMS-D-12-00240.1.

Domingues, R., and Coauthors, 2019: Ocean observations in support of studies and forecasts of tropical and extratropical cyclones. Front. Mar. Sci., 6, 446, https://doi.org/10.3389/ fmars.2019.00446.

Donelan, M. A., B. K. Haus, N. Reul, W. J. Plant, M. Stiassnie, H. C. Graber, O. B. Brown, and E. S. Saltzman, 2004: On the limiting aerodynamic roughness of the ocean in very strong winds. Geophys. Res. Lett., 31, L18306, https://doi.org/10.1029/ 2004GL019460.

Dong, J., and M. Xue, 2013: Assimilation of radial velocity and reflectivity data from coastal WSR-88D radars using an ensemble Kalman filter for the analysis and forecast of landfalling Hurricane Ike (2008). Quart. J. Roy. Meteor. Soc., 139, 467-487, https://doi.org/10.1002/qj.1970.

— , and Coauthors, 2017: Impact of assimilating underwater glider data on Hurricane Gonzalo (2014) forecasts. Wea. Forecasting, 32, 1143-1159, https://doi.org/10.1175/WAF-D-16-0182.1.

Doyle, J., and Coauthors, 2012: Real-time tropical cyclone prediction using COAMPS-TC. Advances in Geosciences, Vol. 28, World Scientific, 15-28.

Evensen, G., 1994: Sequential data assimilation with a nonlinear quasi-geostrophic model using Monte Carlo methods to forecast error statistics. J. Geophys. Res., 99, 10143-10162, https://doi.org/10.1029/94JC00572.

Flaounas, E., and Coauthors, 2018: Assessment of an ensemble of ocean-atmosphere coupled and uncoupled regional climate models to reproduce the climatology of Mediterranean cyclones. Climate Dyn., 51, 1023-1040, https://doi.org/10.1007/ s00382-016-3398-7.

Fortin, V., M. Abaza, F. Anctil, and R. Turcotte, 2014: Why should ensemble spread match the RMSE of the ensemble mean? J. Hydrometeor., 15, 1708-1713, https://doi.org/10.1175/JHMD-14-0008.1.

Frolov, S., C. H. Bishop, T. Holt, J. Cummings, and D. Kuhl, 2016: Facilitating strongly coupled ocean-atmosphere data assimilation with an interface solver. Mon. Wea. Rev., 144, 3-20, https://doi.org/10.1175/MWR-D-15-0041.1.

Fujii, S., and Coauthors, 2013: An overview of developments and applications of oceanographic radar networks in Asia and Oceania countries. Ocean Sci. J., 48, 69-97, https://doi.org/ 10.1007/s12601-013-0007-0.
Gall, R., J. Franklin, F. Marks, E. N. Rappaport, and F. Toepfer, 2013: The Hurricane Forecast Improvement Project. Bull. Amer. Meteor. Soc., 94, 329-343, https://doi.org/10.1175/ BAMS-D-12-00071.1.

Garcia, M., T. Hoar, M. Thomas, B. Bailey, and J. Castillo, 2016: Interfacing an ensemble data assimilation system with a 3D nonhydrostatic Coastal Ocean Model, an OSSE experiment. OCEANS 2016 MTS/IEEE Monterey, Monterey, CA, IEEE, https://doi.org/10.1109/OCEANS.2016.7760992.

Gaspari, G., and S. E. Cohn, 1999: Construction of correlation functions in two and three dimensions. Quart. J. Roy. Meteor. Soc., 125, 723-757, https://doi.org/10.1002/qj.49712555417.

Goni, G., and Coauthors, 2017: Autonomous and Lagrangian ocean observations for Atlantic tropical cyclone studies and forecasts. Oceanography, 30, 92-103, https://doi.org/10.5670/ oceanog.2017.227.

Green, B. W., and F. Zhang, 2013: Impacts of air-sea flux parameterizations on the intensity and structure of tropical cyclones. Mon. Wea. Rev., 141, 2308-2324, https://doi.org/10.1175/ MWR-D-12-00274.1.

Ha, S. Y., and C. Snyder, 2014: Influence of surface observations in mesoscale data assimilation using an ensemble Kalman filter. Mon. Wea. Rev., 142, 1489-1508, https://doi.org/10.1175/ MWR-D-13-00108.1.

Haza, A. C., and Coauthors, 2018: Drogue-loss detection for surface drifters during the Lagrangian Submesoscale Experiment (LASER). J. Atmos. Oceanic Technol., 35, 705-725, https:// doi.org/10.1175/JTECH-D-17-0143.1.

He, J., and Coauthors, 2019: Development and evaluation of an ensemble-based data assimilation system for regional reanalysis over the Tibetan Plateau and surrounding regions. J. Adv. Model. Earth Syst., 11, 2503-2522, https://doi.org/ 10.1029/2019MS001665.

Heming, J. T., 2016: Met Office unified model tropical cyclone performance following major changes to the initialization scheme and a model upgrade. Wea. Forecasting, 31, 14331449, https://doi.org/10.1175/WAF-D-16-0040.1.

Hodur, R. M., 1997: The Naval Research Laboratory's Coupled Ocean/Atmosphere Mesoscale Prediction System (COAMPS). Mon. Wea. Rev., 125, 1414-1430, https://doi.org/10.1175/15200493(1997)125<1414:TNRLSC > 2.0.CO;2.

Holt, T. R., J. A. Cummings, C. H. Bishop, J. D. Doyle, X. Hong, S. Chen, and Y. Jin, 2011: Development and testing of a coupled ocean-atmosphere mesoscale ensemble prediction system. Ocean Dyn., 61, 1937-1954, https://doi.org/10.1007/ s10236-011-0449-9.

Hong, S.-Y., and J.-O. J. Lim, 2006: The WRF single-moment 6-class microphysics scheme (WSM6). Asia-Pac. J. Atmos. Sci., 42, 129-151.

_- Y. Noh, and J. Dudhia, 2006: A new vertical diffusion package with an explicit treatment of entrainment processes. Mon. Wea. Rev., 134, 2318-2341, https://doi.org/10.1175/MWR3199.1.

Hoteit, I., T. Hoar, G. Gopalakrishnan, N. Collins, J. Anderson, B. Cornuelle, A. Köhl, and P. Heimbach, 2013: A MITgcm/ DART ensemble analysis and prediction system with application to the Gulf of Mexico. Dyn. Atmos. Oceans, 63, 1-23, https://doi.org/10.1016/j.dynatmoce.2013.03.002.

Houtekamer, P. L., and H. L. Mitchell, 1998: Data assimilation using an ensemble Kalman filter technique. Mon. Wea. Rev., 126, 796-811, https://doi.org/10.1175/1520-0493(1998)126<0796: DAUAEK $>2.0 . C O ; 2$.

Iacono, M. J., J. S. Delamere, E. J. Mlawer, M. W. Shephard, S. A. Clough, and W. D. Collins, 2008: Radiative forcing by 
long-lived greenhouse gases: Calculations with the AER radiative transfer models. J. Geophys. Res., 113, D13103, https:// doi.org/10.1029/2008JD009944.

Jiménez, P. A., J. Dudhia, J. F. González-Rouco, J. Navarro, J. P. Montávez, and E. García-Bustamante, 2012: A revised scheme for the WRF surface layer formulation. Mon. Wea. Rev., 140, 898-918, https://doi.org/10.1175/MWR-D-11-00056.1.

Kain, J. S., 2004: The Kain-Fritsch convective parameterization: An update. J. Appl. Meteor., 43, 170-181, https://doi.org/ 10.1175/1520-0450(2004)043<0170:TKCPAU>2.0.CO;2.

Karspeck, A. R., and Coauthors, 2018: A global coupled ensemble data assimilation system using the Community Earth System Model and the Data Assimilation Research Testbed. Quart. J. Roy. Meteor. Soc., 144, 2404-2430, https://doi.org/10.1002/ qj.3308.

Kleist, D. T., 2011: Assimilation of tropical cyclone advisory minimum sea level pressure in the NCEP global data assimilation system. Wea. Forecasting, 26, 1085-1091, https://doi.org/ 10.1175/WAF-D-11-00045.1.

Kunii, M., K. Ito, A. Wada, M. Kunii, K. Ito, and A. Wada, 2017: Preliminary test of a data assimilation system with a regional high-resolution atmosphere-ocean coupled model based on an ensemble Kalman filter. Mon. Wea. Rev., 145, 565-581, https://doi.org/10.1175/MWR-D-16-0068.1.

Lahoz, W. A., and P. Schneider, 2014: Data assimilation: Making sense of earth observation. Front. Environ. Sci., 2, 16, https:// doi.org/10.3389/fenvs.2014.00016.

Laloyaux, P., M. Balmaseda, D. Dee, K. Mogensen, and P. Janssen, 2016a: A coupled data assimilation system for climate reanalysis. Quart. J. Roy. Meteor. Soc., 142, 65-78, https:// doi.org/10.1002/qj.2629.

— , J.-N. Thépaut, and D. Dee, 2016b: Impact of scatterometer surface wind data in the ECMWF coupled assimilation system. Mon. Wea. Rev., 144, 1203-1217, https://doi.org/10.1175/ MWR-D-15-0084.1.

— the twentieth century. J. Adv. Model. Earth Syst., 10, 11721195, https://doi.org/10.1029/2018MS001273.

Larson, J., R. Jacob, and E. Ong, 2005: The model coupling toolkit: A new Fortran90 toolkit for building multiphysics parallel coupled models. Int. J. High Perform. Comput. Appl., 19, 277292, https://doi.org/10.1177/1094342005056115.

Leeuwenburgh, O., 2005: Assimilation of along-track altimeter data in the tropical Pacific region of a global OGCM ensemble. Quart. J. Roy. Meteor. Soc., 131, 2455-2472, https:// doi.org/10.1256/qj.04.146.

Lengaigne, M., and Coauthors, 2019: Influence of air-sea coupling on Indian Ocean tropical cyclones. Climate Dyn., 52, 577-598, https://doi.org/10.1007/s00382-018-4152-0.

Leroux, M.-D., and Coauthors, 2018: Recent advances in research and forecasting of tropical cyclone track, intensity, and structure at landfall. Trop. Cyclone Res. Rev., 7, 85-105, https://doi.org/10.6057/2018TCRR02.02.

Li, J., and H. Liu, 2009: Improved hurricane track and intensity forecast using single field-of-view advanced IR sounding measurements. Geophys. Res. Lett., 36, L11813, https:// doi.org/10.1029/2009GL038285.

Li, L., and Coauthors, 2018: Field survey of Typhoon Hato (2017) and a comparison with storm surge modeling in Macau. Nat. Hazards Earth Syst. Sci., 18, 3167-3178, https://doi.org/ 10.5194/nhess-18-3167-2018.

Li, Y., and R. Toumi, 2017: A balanced Kalman filter ocean data assimilation system with application to the South Australian
Sea. Ocean Modell., 116, 159-172, https://doi.org/10.1016/ j.ocemod.2017.06.007.

— by assimilating coastal surface currents in an idealized study. Geophys. Res. Lett., 45, 10 019-10 026, https://doi.org/10.1029/ 2018 GL079677.

Liu, H., and J. Li, 2010: An improvement in forecasting rapid intensification of Typhoon Sinlaku (2008) using clear-sky full spatial resolution advanced IR Soundings. J. Appl. Meteor. Climatol., 49, 821-827, https://doi.org/10.1175/2009JAMC2374.1.

Liu, Z., C. S. Schwartz, C. Snyder, and S. Y. Ha, 2012: Impact of assimilating AMSU-A radiances on forecasts of 2008 Atlantic tropical cyclones initialized with a limited-area ensemble Kalman filter. Mon. Wea. Rev., 140, 4017-4034, https://doi.org/ 10.1175/MWR-D-12-00083.1.

Ma, L. M., and Z. M. Tan, 2009: Improving the behavior of the cumulus parameterization for tropical cyclone prediction: Convection trigger. Atmos. Res., 92, 190-211, https://doi.org/ 10.1016/j.atmosres.2008.09.022.

Magnusson, L., and Coauthors, 2019: ECMWF activities for improved hurricane forecasts. Bull. Amer. Meteor. Soc., 100, 445-458, https://doi.org/10.1175/BAMS-D-18-0044.1.

Mantovani, C., and Coauthors, 2020: Best practices on high frequency radar deployment and operation for ocean current measurement. Front. Mar. Sci., 7, 210, https://doi.org/10.3389/ fmars.2020.00210.

Meng, Z., and F. Zhang, 2011: Limited-area ensemble-based data assimilation. Mon. Wea. Rev., 139, 2025-2045, https://doi.org/ 10.1175/2011MWR3418.1.

Mogensen, K. S., L. Magnusson, and J.-R. Bidlot, 2017: Tropical cyclone sensitivity to ocean coupling in the ECMWF coupled model. J. Geophys. Res. Oceans, 122, 4392-4412, https:// doi.org/10.1002/2017JC012753.

Molcard, A., L. I. Piterbarg, A. Griffa, T. M. Özgökmen, and A. J. Mariano, 2003: Assimilation of drifter observations for the reconstruction of the Eulerian circulation field. J. Geophys. Res., 108, 3056, https://doi.org/10.1029/2001JC001240.

— A. Ariffa, and T. M. Özgökmen, 2005: Lagrangian data assimilation in multilayer primitive equation ocean models. J. Atmos. Oceanic Technol., 22, 70-83, https://doi.org/10.1175/ JTECH-1686.1.

Muscarella, P., M. J. Carrier, H. Ngodock, S. Smith, B. L. Lipphardt, A. D. Kirwan, and H. S. Huntley, 2015: Do assimilated drifter velocities improve Lagrangian predictability in an operational ocean model? Mon. Wea. Rev., 143, 18221832, https://doi.org/10.1175/MWR-D-14-00164.1.

Na, L., L. Tiejun, W. Hui, Z. Yunfei, G. Zhiyi, and W. Yi, 2015: Numerical simulation of Typhoon Muifa (2011) using a Coupled Ocean-Atmosphere-Wave-Sediment Transport (COAWST) modeling system. J. Ocean Univ. China, 14, 199-209, https://doi.org/10.1007/s11802-015-2415-5.

National Centers for Environmental Prediction/National Weather Service/NOAA/U.S. Department of Commerce, 2015: NCEP GFS 0.25 Degree Global Forecast Grids Historical Archive. Research Data Archive at the National Center for Atmospheric Research, Computational and Information Systems Laboratory, Boulder, CO, accessed 11 October 2018, https://doi.org/10.5065/ D65D8PWK.

Neumann, B., A. T. Vafeidis, J. Zimmermann, and R. J. Nicholls, 2015: Future coastal population growth and exposure to sea-level rise and coastal flooding-A global assessment. PLOS One, 10, e0131375, https://doi.org/10.1371/ journal.pone.0118571. 
Nilsson, J. A. U., S. Dobricic, N. Pinardi, P.-M. Poulain, and D. Pettenuzzo, 2012: Variational assimilation of Lagrangian trajectories in the Mediterranean Ocean forecasting system. Ocean Sci., 8, 249-259, https://doi.org/10.5194/os-8-249-2012.

NOAA Office of Ocean Exploration and Research, 2005: Physical Oceanography Division (PhOD) -Global Drifter Program. NOAA, accessed 10 September 2020, https:// www.aoml.noaa.gov/phod/gdp.

Nodet, M., 2006: Variational assimilation of Lagrangian data in oceanography. Inverse Probl., 22, 245-263, https://doi.org/ 10.1088/0266-5611/22/1/014.

Novelli, G., C. M. Guigand, C. Cousin, E. H. Ryan, N. J. Laxague, H. Dai, B. K. Haus, and T. M. Özgökmen, 2017: A biodegradable surface drifter for ocean sampling on a massive scale. J. Atmos. Oceanic Technol., 34, 2509-2532, https://doi.org/ 10.1175/JTECH-D-17-0055.1.

Özgökmen, T. M., A. Molcard, T. M. Chin, L. I. Piterbarg, and A. Griffa, 2003: Assimilation of drifter observations in primitive equation models of midlatitude ocean circulation. J. Geophys. Res., 108, 3238, https://doi.org/10.1029/2002JC001719.

Paduan, J. D., and L. Washburn, 2013: High-frequency radar observations of ocean surface currents. Annu. Rev. Mar. Sci., 5, 115136, https://doi.org/10.1146/annurev-marine-121211-172315.

Penny, S. G., and T. M. Hamill, 2017: Coupled data assimilation for integrated earth system analysis and prediction. Bull. Amer. Meteor. Soc., 98, ES169-ES172, https://doi.org/10.1175/ BAMS-D-17-0036.1.

—, D. W. Behringer, J. A. Carton, and E. Kalnay, 2015: A hybrid Global Ocean Data Assimilation System at NCEP. Mon. Wea. Rev., 143, 4660-4677, https://doi.org/10.1175/MWR-D-14-00376.1.

—, E. Bach, K. Bhargava, C. Chang, C. Da, L. Sun, and T. Yoshida, 2019: Strongly coupled data assimilation in multiscale media: Experiments using a quasi-geostrophic coupled model. J. Adv. Model. Earth Syst., 11, 1803-1829, https:// doi.org/10.1029/2019MS001652.

Phillipson, L., and R. Toumi, 2017: Impact of data assimilation on ocean current forecasts in the Angola Basin. Ocean Modell., 114, 45-58, https://doi.org/10.1016/j.ocemod.2017.04.006.

Poje, A. C., M. Toner, A. D. Kirwan Jr., and C. K. R. T. Jones, 2002: Drifter launch strategies based on Lagrangian templates. J. Phys. Oceanogr., 32, 1855-1869, https://doi.org/10.1175/ 1520-0485(2002)032<1855:DLSBOL > 2.0.CO;2.

Price, J. F., 1981: Upper ocean response to a hurricane. J. Phys. Oceanogr., 11, 153-175, https://doi.org/10.1175/1520-0485(1981) 011<0153:UORTAH > 2.0.CO;2.

Pu, Z., X. Li, C. S. Velden, S. D. Aberson, and W. T. Liu, 2008: The impact of aircraft dropsonde and satellite wind data on numerical simulations of two landfalling tropical storms during the Tropical Cloud Systems and Processes experiment. Wea. Forecasting, 23, 62-79, https://doi.org/10.1175/ 2007WAF2007006.1.

$\longrightarrow$ - _ a and J. Sun, 2009: Impact of airborne Doppler radar data assimilation on the numerical simulation of intensity changes of Hurricane Dennis near a landfall. J. Atmos. Sci., 66, 33513365, https://doi.org/10.1175/2009JAS3121.1.

— C. Yu, V. Tallapragada, J. Jin, and W. Mccarty, 2019: The impact of assimilation of GPM microwave imager clear-sky radiance on numerical simulations of Hurricanes Joaquin (2015) and Matthew (2016) with the HWRF Model. Mon. Wea. Rev., 147, 175-198, https://doi.org/10.1175/MWR-D-17-0200.1.

Pun, I.-F., and Coauthors, 2019: Rapid intensification of Typhoon Hato (2017) over shallow water. Sustainability, 11, 3709, https://doi.org/10.3390/su11133709.
Raeder, K., J. L. Anderson, N. Collins, T. J. Hoar, J. E. Kay, P. H. Lauritzen, and R. Pincus, 2012: DART/CAM: An ensemble data assimilation system for CESM atmospheric models. J. Climate, 25, 6304-6317, https://doi.org/10.1175/JCLI-D-1100395.1.

Roarty, H., and Coauthors, 2019: The global high frequency radar network. Front. Mar. Sci., 6, 164, https://doi.org/10.3389/ fmars.2019.00164.

Romine, G. S., C. S. Schwartz, C. Snyder, J. L. Anderson, and M. L. Weisman, 2013: Model bias in a continuously cycled assimilation system and its influence on convection-permitting forecasts. Mon. Wea. Rev., 141, 1263-1284, https://doi.org/ 10.1175/MWR-D-12-00112.1.

Saha, S., and Coauthors, 2006: The NCEP Climate Forecast System. J. Climate, 19, 3483-3517, https://doi.org/10.1175/JCLI3812.1.

Salman, H., L. Kuznetsov, C. K. R. T. Jones, and K. Ide, 2006: A method for assimilating Lagrangian data into a shallow-waterequation ocean model. Mon. Wea. Rev., 134, 1081-1101, https://doi.org/10.1175/MWR3104.1.

Sampson, C. R., E. M. Fukada, J. A. Knaff, B. R. Strahl, M. J. Brennan, and T. Marchok, 2017: Tropical cyclone gale wind radii estimates for the western North Pacific. Wea. Forecasting, 32, 1029-1040, https://doi.org/10.1175/WAF-D-16-0196.1.

Sandery, P. A., G. B. Brassington, A. Craig, and T. Pugh, 2010: Impacts of ocean-atmosphere coupling on tropical cyclone intensity change and ocean prediction in the Australian region. Mon. Wea. Rev., 138, 2074-2091, https://doi.org/10.1175/ 2010MWR3101.1.

Sanford, T. B., P. G. Black, J. R. Haustein, J. W. Feeney, G. Z. Forristall, and J. F. Price, 1987: Ocean response to a Hurricane. Part I: Observations. J. Phys. Oceanogr., 17, 2065-2083, https://doi.org/10.1175/1520-0485(1987)017<2065: ORTAHP $>2.0 . \mathrm{CO} ; 2$.

Shchepetkin, A. F., and J. C. McWilliams, 2005: The Regional Oceanic Modeling System (ROMS): A split-explicit, freesurface, topography-following-coordinate oceanic model. Ocean Modell., 9, 347-404, https://doi.org/10.1016/j.ocemod.2004.08.002.

Shen, Y.-T., and Coauthors, 2019: Applications of ocean currents data from high-frequency radars and current profilers to search and rescue missions around Taiwan. J. Oper. Oceanogr., 12, S126S136, https://doi.org/10.1080/1755876X.2018.1541538.

Singh, T., 2020: Development of an ensemble data assimilation system with LMDZ5 AGCM for regional reanalysis. Climate Dyn., 54, 2847-2868, https://doi.org/10.1007/s00382020-05147-z.

Skamarock, W. C., and Coauthors, 2008: A description of the Advanced Research WRF version 3. NCAR Tech. Note NCAR/ TN-475+STR, 113 pp., https://doi.org/10.5065/D68S4MVH..

Sluka, T. C., S. G. Penny, E. Kalnay, and T. Miyoshi, 2016: Assimilating atmospheric observations into the ocean using strongly coupled ensemble data assimilation. Geophys. Res. Lett., 43, 752-759, https://doi.org/10.1002/2015GL067238.

Smith, P. J., A. M. Fowler, and A. S. Lawless, 2015: Exploring strategies for coupled 4D-Var data assimilation using an idealised atmosphere-ocean model. Tellus, 67A, 27025, https:// doi.org/10.3402/tellusa.v67.27025.

_ A. S. Lawless, and N. K. Nichols, 2018: Treating sample covariances for use in strongly coupled atmosphere-ocean data assimilation. Geophys. Res. Lett., 45, 445-454, https://doi.org/ 10.1002/2017GL075534.

Srinivas, C. V., G. M. Mohan, C. V. Naidu, R. Baskaran, and B. Venkatraman, 2016: Impact of air-sea coupling on the simulation of tropical cyclones in the North Indian Ocean using a 
simple 3-D ocean model coupled to ARW. J. Geophys. Res. Atmos., 121, 9400-9421, https://doi.org/10.1002/2015JD024431.

Sun, L., and S. G. Penny, 2019: Lagrangian data assimilation of surface drifters in a double-gyre ocean model using the local ensemble transform Kalman filter. Mon. Wea. Rev., 147, 45334551, https://doi.org/10.1175/MWR-D-18-0406.1.

Talagrand, O., and P. Courtier, 1987: Variational assimilation of meteorological observations with the adjoint vorticity equation. I: Theory. Quart. J. Roy. Meteor. Soc., 113, 1311-1328, https://doi.org/10.1002/qj.49711347812.

Ting, M., J. P. Kossin, S. J. Camargo, and C. Li, 2019: Past and future hurricane intensity change along the U.S. East coast. Sci. Rep., 9, 7795, https://doi.org/10.1038/s41598-019-44252-w.

Torn, R. D., and G. J. Hakim, 2008: Performance characteristics of a pseudo-operational ensemble Kalman filter. Mon. Wea. Rev., 136, 3947-3963, https://doi.org/10.1175/ 2008MWR2443.1.

——, and — 2009: Ensemble data assimilation applied to RAINEX observations of Hurricane Katrina (2005). Mon. Wea. Rev., 137, 2817-2829, https://doi.org/10.1175/ 2009MWR2656.1.

Wan, L., J. Zhu, L. Bertino, and H. Wang, 2008: Initial ensemble generation and validation for ocean data assimilation using HYCOM in the Pacific. Ocean Dyn., 58, 81-99, https://doi.org/ 10.1007/s10236-008-0133-x.

Wang, S., and R. Toumi, 2018: A historical analysis of the mature stage of tropical cyclones. Int. J. Climatol., 38, 2490-2505, https://doi.org/10.1002/joc.5374.

Warner, J. C., B. Armstrong, R. He, and J. B. Zambon, 2010: Development of a Coupled Ocean-Atmosphere-WaveSediment Transport (COAWST) Modeling System. Ocean Modell., 35, 230-244, https://doi.org/10.1016/j.ocemod.2010. 07.010 .

Weng, Y., and F. Zhang, 2012: Assimilating airborne Doppler radar observations with an ensemble Kalman filter for convection-permitting hurricane initialization and prediction: Katrina (2005). Mon. Wea. Rev., 140, 841-859, https://doi.org/ 10.1175/2011MWR3602.1.

Wilks, D. S., 2011: On the reliability of the rank histogram. Mon. Wea. Rev., 139, 311-316, https://doi.org/10.1175/2010MWR3446.1.

World Bank, 2015: East Asia's Changing Urban Landscape: Measuring a Decade of Spatial Growth. The World Bank, 180 pp., https://doi.org/10.1596/978-1-4648-0363-5.

Yang, C., Z. Liu, J. Bresch, S. R. H. Rizvi, X.-Y. Huang, and J. Min, 2016: AMSR2 all-sky radiance assimilation and its impact on the analysis and forecast of Hurricane Sandy with a limited-area data assimilation system. Tellus, 68A, 30917, https://doi.org/ 10.3402/tellusa.v68.30917.

Yihong, D., W. Rongsheng, Y. Runling, and L. Xudong, 2013: Numerical simulation of changes in tropical cyclone intensity using a coupled air-sea model. Acta Meteor. Sin., 27, 658-672, https://doi.org/10.1007/s13351-013-0503-2.

Yoshida, T., and E. Kalnay, 2018: Correlation-cutoff method for covariance localization in strongly coupled data assimilation. Mon. Wea. Rev., 146, 2881-2889, https://doi.org/10.1175/ MWR-D-17-0365.1.

Zambon, J. B., R. He, and J. C. Warner, 2014: Investigation of Hurricane Ivan using the Coupled Ocean-Atmosphere-WaveSediment Transport (COAWST) model. Ocean Dyn., 64, 1535-1554, https://doi.org/10.1007/s10236-014-0777-7.

Zhang, F., and Y. Weng, 2015: Predicting hurricane intensity and associated hazards: A five-year real-time forecast experiment with assimilation of airborne Doppler radar observations. Bull. Amer. Meteor. Soc., 96, 25-33, https://doi.org/10.1175/ BAMS-D-13-00231.1.

— , and K. Emanuel, 2018: Promises in air-sea fully coupled data assimilation for future hurricane prediction. Geophys. Res. Lett., 45, 13 173-13 177, https://doi.org/10.1029/2018GL080970.

—, Y. Weng, J. A. Sippel, Z. Meng, and C. H. Bishop, 2009: Cloud-resolving hurricane initialization and prediction through assimilation of Doppler radar observations with an ensemble Kalman filter. Mon. Wea. Rev., 137, 2105-2125, https://doi.org/10.1175/2009MWR2645.1.

Zhang, H., Z. Pu, H. Zhang, and Z. Pu, 2014: Influence of assimilating surface observations on numerical prediction of landfalls of Hurricane Katrina (2005) with an ensemble Kalman filter. Mon. Wea. Rev., 142, 2915-2934, https://doi.org/10.1175/ MWR-D-14-00014.1.

Zhang, S., M. J. Harrison, A. Rosati, and A. Wittenberg, 2007: System design and evaluation of coupled ensemble data assimilation for global oceanic climate studies. Mon. Wea. Rev., 135, 3541-3564, https://doi.org/10.1175/MWR3466.1.

_ , and Coauthors, 2020: Coupled data assimilation and parameter estimation in coupled ocean-atmosphere models: A review. Climate Dyn., 54, 5127-5144, https://doi.org/10.1007/s00382-02005275-6.

Zhao, L., Y. Chen, and V. S. Sheng, 2020: A real-time typhoon eye detection method based on deep learning for meteorological information forensics. J. Real-Time Image Process., 17, 95102, https://doi.org/10.1007/s11554-019-00899-2. 